\title{
Motion of flux transfer events: a test of the Cooling model
}

\author{
R. C. Fear ${ }^{1}$, S. E. Milan ${ }^{1}$, A. N. Fazakerley ${ }^{2}$, C. J. Owen ${ }^{2}$, T. Asikainen ${ }^{3}$, M. G. G. T. Taylor ${ }^{4}$, E. A. Lucek ${ }^{5}$, H. Rème ${ }^{6}$, \\ I. Dandouras ${ }^{6}$, and P. W. Daly ${ }^{7}$ \\ ${ }^{1}$ Department of Physics and Astronomy, University of Leicester, Leicester, LE1 7RH, UK \\ ${ }^{2}$ Mullard Space Science Laboratory, University College London, Holmbury St. Mary, Dorking, Surrey, RH5 6NT, UK \\ ${ }^{3}$ Department of Physical Sciences, University of Oulu, P.O. Box 3000, 90014, Finland \\ ${ }^{4}$ Research and Scientific Support Department, European Space Agency, 2201AZ Noordwijk, The Netherlands \\ 5 Blackett Laboratory, Imperial College, Prince Consort Road, London, SW7 2BZ, UK \\ ${ }^{6}$ CESR/CNRS, 9 Avenue du Colonel Roche, B.P. 4346, 31028 Toulouse Cedex 4, France \\ ${ }^{7}$ Max-Planck-Institut für Sonnensystemforschung, Max-Planck-Str. 2, 37191 Katlenburg-Lindau, Germany
}

Received: 30 March 2007 - Revised: 29 June 2007 - Accepted: 13 July 2007 - Published: 30 July 2007

\begin{abstract}
The simple model of reconnected field line motion developed by Cooling et al. (2001) has been used in several recent case studies to explain the motion of flux transfer events across the magnetopause. We examine 213 FTEs observed by all four Cluster spacecraft under a variety of IMF conditions between November 2002 and June 2003, when the spacecraft tetrahedron separation was $\sim 5000 \mathrm{~km}$. Observed velocities were calculated from multi-spacecraft timing analysis, and compared with the velocities predicted by the Cooling model in order to check the validity of the model. After excluding three categories of FTEs (events with poorly defined velocities, a significant velocity component out of the magnetopause surface, or a scale size of less than $5000 \mathrm{~km}$ ), we were left with a sample of 118 events. $78 \%$ of these events were consistent in both direction of motion and speed with one of the two model de Hoffmann-Teller (dHT) velocities calculated from the Cooling model (to within $30^{\circ}$ and a factor of two in the speed). We also examined the plasma signatures of several magnetosheath FTEs; the electron signatures confirm the hemisphere of connection indicated by the model in most cases. This indicates that although the model is a simple one, it is a useful tool for identifying the source regions of FTEs.
\end{abstract}

Keywords. Magnetospheric physics (Magnetopause, cusp, arid boundary layers; Solar wind-magnetosphere interactions) - Space plasma physics (Magnetic reconnection)

Correspondence to: R. C. Fear

(r.fear@ion.le.ac.uk)

\section{Introduction}

Magnetic reconnection at the Earth's magnetopause (Dungey, 1961, 1963) is the dominant process of energy and momentum transfer from the solar wind into the terrestrial magnetosphere (Cowley, 1984). The basic process of reconnection as envisaged by Dungey $(1961,1963)$ was steady state. Indeed, reconnection may occur as a continuous process for hours at a time (Frey et al., 2003; Phan et al., 2004). However, magnetopause reconnection commonly occurs as a transient or time-varying process, as first observed by Haerendel et al. (1978) in the form of signatures they called "flux erosion events".

Independently, Russell and Elphic $(1978,1979)$ identified signatures of transient reconnection at the low-latitude magnetopause which they called "flux transfer events" (FTEs). These signatures were interpreted as open flux ropes, formed by reconnection, which moved away from the reconnection site under the net effect of the force exerted by the solar wind flow and the $\boldsymbol{j} \times \boldsymbol{B}$ magnetic tension force. They were identified by inspecting the magnetic field data in the magnetosheath or magnetosphere in a boundary normal coordinate system (introduced by Russell and Elphic, 1978), in which $\hat{N}$ is normal to the magnetopause and directed away from Earth. An FTE exhibits a bipolar signature in the $B_{N}$ component. In the simplest case, the bipolar signature is formed by the draping of unreconnected magnetic field lines around the FTE. The flux erosion events reported by Haerendel et al. (1978) were shown to be flux transfer events by Rijnbeek and Cowley (1984).

The polarity of the $B_{N}$ signature depends upon the motion of the FTE relative to the unperturbed magnetic field. In the magnetosheath, a "standard" or "direct" signature (a

Published by Copernicus Publications on behalf of the European Geosciences Union. 
positive followed by a negative deflection in $B_{N}$ ) occurs if the FTE velocity has a component antiparallel to the local magnetosheath magnetic field, whereas a "reverse" signature (negative/positive) occurs if the velocity has a component parallel to the magnetic field (Rijnbeek et al., 1982). If the FTE is observed from inside the magnetosphere, a standard signature occurs if the FTE velocity has a component parallel to the geomagnetic field and a reverse component if the component is antiparallel. There is often a significant increase or decrease in $|\boldsymbol{B}|$, and an imbalance in the total pressure $\left(p_{\text {gas }}+B^{2} / 2 \mu_{0}\right)$ countered by the magnetic tension in the draped magnetic field lines (Paschmann et al., 1982). A bipolar $B_{N}$ signature is also observed when the reconnected flux tube was crossed, explained by a helicity in the reconnected flux rope (Sonnerup, 1987).

Several alternative reconnection-based models have been proposed which explain the observations: Lee and $\mathrm{Fu}$ (1985) proposed a model where helical flux tubes were generated by multiple reconnection lines (X-lines). Southwood et al. (1988) and Scholer (1988) independently proposed a model based on a single $\mathrm{X}$-line, where the magnetopause boundary layer thickens and then thins as a result of a variation in the reconnection rate, producing a bulge which propagates under the same magnetosheath flow and $\boldsymbol{j} \times \boldsymbol{B}$ effects. This two dimensional model does not produce a tube of reconnected flux, but can extend a considerable distance along the magnetopause.

It has also been suggested that FTE-style signatures can be formed by magnetopause waves, although this has been hotly debated (e.g. Sibeck et al., 1989; Lanzerotti, 1989; Sibeck, 1990; Elphic, 1990; Sckopke, 1991; Lockwood, 1991; Sibeck, 1992; Smith and Owen, 1992; Kawano et al., 1992; Elphic et al., 1994; Song et al., 1994, 1996; Sibeck and Newell, 1995, 1996; Sanny et al., 1996). In the context of this debate, Kawano et al. (1992) introduced a "characteristic time" $\left(t_{\text {char }}\right.$, defined as the time between the positive and negative peaks in the $B_{N}$ signature) to distinguish between longer events with bipolar $B_{N}$ signatures $\left(t_{\text {char }}>90 \mathrm{~s}\right)$ which were found to occur over a wide range of McIlwain L-shells and were not correlated to periods of reconnection as evidenced by AE index or southward interplanetary magnetic field (IMF), and shorter events $\left(t_{\text {char }}<90 \mathrm{~s}\right)$ which occurred nearer the magnetopause during periods of high AE index and southward IMF. Sanny et al. (1996) investigated similar signatures, over a wider range of radial distances from Earth and also concluded that the shorter events were FTEs, whilst the longer events were more likely to be due to magnetopause waves.

Much work has been done on the statistics of FTE occurrence. Early surveys (Berchem and Russell, 1984; Rijnbeek et al., 1984; Southwood et al., 1986; Kuo et al., 1995) examined the pre-terminator magnetopause $\left(X_{\mathrm{GSM}}>0\right)$, and found that FTEs were strongly correlated with southward IMF, consistent with low-latitude reconnection as a source of FTEs. Standard polarity FTEs are generally observed in the North- ern Hemisphere, whilst reverse polarity FTEs are generally observed in the Southern Hemisphere (Rijnbeek et al., 1984), although the division between these events is often inclined to the magnetic equator (Berchem and Russell, 1984). Russell et al. (1985) showed the polarity, and hence motion, of FTE signatures which occurred when the IMF was southward to be consistent with low-latitude reconnection even when there is a dominant IMF $B_{Y}$ component.

More recent surveys have extended to the post-terminator region. Kawano and Russell (1997a,b) studied a database of 1246 FTEs, of which 79 occurred in the post-terminator region $\left(X_{\mathrm{GSM}}<0,\left|Z_{\mathrm{GSM}}\right|<15 R_{E}\right)$ when the IMF was northward. It was proposed that most of these events could be explained by a tilted, subsolar component reconnection line if open flux tubes in the subsolar region were immediately closed by a process of "re-reconnection" (Nishida, 1989), thus preventing northward IMF FTEs from being observed in the subsolar region. When the IMF was more strongly northward, Kawano and Russell (1997b) concluded that the polarities and IMF $B_{Y}$ dependency could also be explained if the FTEs were generated near the polar cusps at an antiparallel reconnection site, but then somehow moved equatorward and tailward.

The anisotropy of plasma signatures associated with magnetosheath FTEs can be used to determine the hemisphere of connection of an FTE. Plasma populations originating from the magnetosphere and with a field-aligned anisotropy were first observed inside magnetosheath FTEs by Daly et al. (1981), consistent with the spacecraft being on open, reconnected magnetic field lines. A parallel beam observed on open magnetic field lines in the magnetosheath implies a connection to the Southern Hemisphere, whereas an antiparallel beam implies connection to the Northern Hemisphere. (A plasma signature is also observed in magnetospheric FTEs, e.g. Paschmann et al. (1982), although the scenario is complicated by the mirroring of plasma at the ionosphere.) Furthermore, Daly et al. (1984) found that there was sometimes an inconsistency between the direction of motion inferred from the FTE polarity (standard or reverse) and the anisotropy of high-energy ion signatures (above $25 \mathrm{keV}$ ). This inconsistency could be resolved if FTEs were generated in regions where the magnetosheath flow was super-Alfvénic; accordingly a flux tube connected to the Southern Hemisphere but crossing the magnetopause in the Northern Hemisphere could be dragged northward if the force exerted by the magnetosheath flow was stronger than the southward-directed magnetic tension. Daly et al. (1984) also noted that magnetosheath FTEs commonly exhibited an increase in intensity of electrons above $20 \mathrm{keV}$, although not usually to magnetospheric levels, but that these signatures were commonly isotropic. It was concluded that the lower intensity levels were due to the rapid depopulation of high-energy magnetospheric electrons once a field line had been opened by reconnection. The isotropy of the electron signatures (and small enhancements in the ion intensities in the opposite 
direction to the main enhancement) was attributed to electromagnetic waves associated with FTEs (Anderson et al., 1982). More recently, Robert et al. (2005) studied a magnetosheath FTE which was associated with a bidirectional electron signature at lower energies (accelerated magnetosheath electrons, rather than escaping magnetospheric electrons); however, these signatures were interpreted as an entry onto closed magnetospheric field lines within the core of the FTE.

The launch of the Cluster spacecraft has enabled fourpoint observations of flux transfer events for the first time (Owen et al., 2001), which allows the velocity of FTEs to be determined by multi-spacecraft timing analysis (Russell et al., 1983; Harvey, 1998). Wild et al. (2005) and Dunlop et al. (2005) have calculated the velocities of a small number of FTEs in this way, and compared them with a model of reconnected field line motion developed by Cooling et al. (2001). Fear et al. (2005b) identified 446 FTEs observed in a survey of one season in which the Cluster spacecraft crossed the magnetopause (November 2002-June 2003). This survey included 120 FTEs which occurred when the lagged IMF was strongly northward (defined as when the magnitude of the IMF clock angle $\left(\theta_{C A}=\arctan \left(B_{Y} / B_{Z}\right)\right)$ was less than $70^{\circ}$ ), which were mostly in the Southern Hemisphere and in the post-terminator region. Many of the events which occurred at lower latitudes had a slight equatorward velocity. The equatorward motion (one of the possibilities proposed by Kawano and Russell, 1997b) was explained by the Cooling et al. (2001) model, which showed that if the component reconnection site was initiated in the Southern Hemisphere and in the super-Alfvénic magnetosheath flow region, the open magnetic field lines which were connected to the Southern Hemisphere ionosphere would be swept across the location of the X-line and could be swept equatorward, matching the observed motion of the FTEs.

Wang et al. $(2005,2006)$ presented a larger survey of 1222 FTEs observed by Cluster, based on two and a half magnetopause crossing seasons (February 2001 to June 2003). These authors observed that approximately $30 \%$ of events were observed by all four spacecraft, although during this time the inter-spacecraft distance at the magnetopause crossing varied from $\sim 100 \mathrm{~km}$ to $\sim 1 R_{E}$. Furthermore, approximately $73 \%$ of events were observed in the magnetosheath, as opposed to being observed in the cusp region or the magnetosphere-proper (an observation also made by Fear et al., 2005b). A similar percentage of magnetosheath events was observed if the high latitude (near-noon) and low latitude (flank) regions were examined separately. Wang et al. (2005) suggested that this was because FTEs were more likely to extend further out into the magnetosheath than they extend into the magnetosphere; recent analysis of some individual FTEs observed by Cluster has confirmed that this may be the case (Hasegawa et al., 2006). However, a further possible explanation is that the magnetospheric signatures of FTEs at high latitudes, particularly in the vicinity of the cusp, may often be more complicated than low latitude signatures, possibly leading to an underestimate of the number of magnetospheric events (Thompson et al., 2004; Fear et al., 2005a).

In this paper, we seek to determine the reliability of the Cooling et al. (2001) model of reconnected field line motion (hereinafter referred to as the "Cooling model"). In Sect. 2, we discuss the instrumentation and event catalogue to be used in this paper. Then, we introduce the multi-spacecraft timing analysis technique used to determine FTE velocities, and the method to ensure the robustness of these results in Sect. 3. The Cooling model is discussed in Sect. 4, and a case-by-case comparison of the determined FTE velocities and the model is made in Sect. 5. The plasma signatures of FTEs are investigated in Sect. 6. The reliability of the model is discussed in Sect. 7, and the results are summarised in Sect. 8.

\section{Data set and instrumentation}

In this study, we use data from the Cluster FGM (Balogh et al., 2001), PEACE (Johnstone et al., 1997), CIS (Rème et al., 2001) and RAPID (Wilken et al., 2001) instruments. PEACE provides observations of electrons at energies below $26.4 \mathrm{keV}$, and CIS observes low-energy ions $(\mathrm{E}<32 \mathrm{keV}$ and $\mathrm{E}<40 \mathrm{keV}$ for the HIA and CODIF sensors respectively). RAPID complements these instruments with observations of higher-energy electrons and ions (above 37 and $27 \mathrm{keV}$, respectively). In this paper, we use $5 \mathrm{~Hz}$ observations of the magnetosheath/magnetospheric magnetic field provided by FGM. The solar wind conditions are provided by the ACE spacecraft. The lagged IMF was calculated using $64 \mathrm{~s}$ averages of the solar wind speed from the SWEPAM instrument (McComas et al., 1998) and 4 min averages of the IMF from the MAG instrument (Smith et al., 1998).

We use the catalogue of 446 FTEs compiled by Fear et al. (2005b). FTEs were selected for this catalogue if both a clear bipolar signature in $B_{N}$ and a change (enhancement or decrease) in $|B|$ were observed by at least one spacecraft. The change in $|B|$ had to be centred close to the centre of the $B_{N}$ signature. FTEs were excluded if they coincided with a magnetopause crossing at all spacecraft which observed the $B_{N}$ signature (i.e. magnetosphere-FTE-magnetosheath, or magnetosheath-FTE-magnetosphere).

The catalogue extends from November 2002 to June 2003. In this period, the orbit of the Cluster spacecraft precessed such that it crossed the low-latitude magnetopause on the dusk flank (November/December 2002), through the highlatitude magnetopause at local times near noon (JanuaryApril 2003), and finally the low-latitude magnetopause on the dawn flank (May/June 2003). During this season, the separation of the Cluster spacecraft was of the order of $1 R_{E}$, which is the scale size of an FTE normal to the magnetopause as determined by low-latitude observations (Saunders et al., 1984). This separation is useful for studying the velocities of 


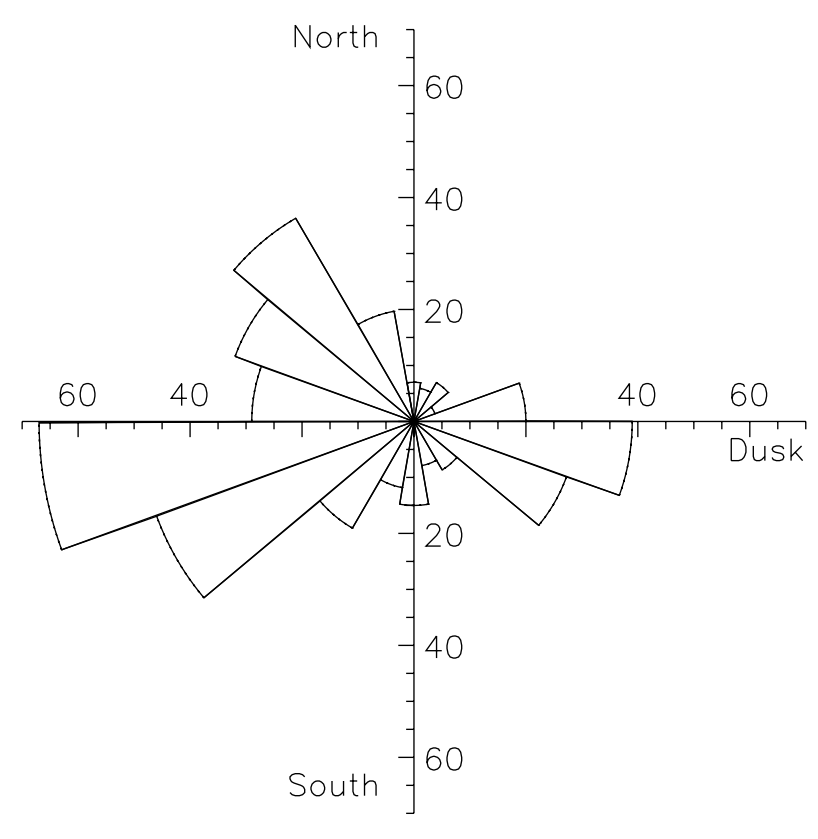

Fig. 1. A polar histogram of the lagged IMF clock angle at the time of observation of each FTE. The angle clockwise from north is the IMF clock angle; hence the angles are those as viewed from the Sun. North and south $\left( \pm Z_{\mathrm{GSM}}\right)$ are at the top and bottom, and dusk \& dawn $\left( \pm Y_{\mathrm{GSM}}\right)$ are right $\&$ left. Each bin is $20^{\circ}$ wide.

FTEs. If the separation were smaller, only a small time difference would be observed between the signatures at the different spacecraft leading to a larger uncertainty in determining FTE motion. On the other hand, if it were larger it is unlikely that many events would be observed by all four spacecraft, rendering velocity determination by multi-spacecraft timing impossible.

A histogram of the dependence of FTE occurrence on IMF clock angle is shown in Fig. 1. The solar wind lag time was determined for each FTE by calculating the arrival time of each 4-min parcel of solar wind plasma in the three hours before each FTE using $V_{\text {XGSM }}$ observed by ACE and the separation in $X_{\mathrm{GSM}}$ between ACE and Cluster 3. The lagged IMF was plotted with the magnetosheath magnetic field observed by Cluster, and the lag was adjusted by eye where necessary (Fear et al., 2005b). Most FTEs were observed when the IMF was southward but strongly dawnward or duskward. This is consequence of the orbit of Cluster. Since one would expect FTEs formed in the subsolar regions during intervals of low IMF $B_{Y}$ to move predominantly latitudinally, whereas those formed under $B_{Y}$-dominated IMF would move more longitudinally, there is a higher probability of observing $B_{Y}$-dominated events on the flanks than at the high-latitude, near-noon magnetopause. The Cluster spacecraft spend relatively little time near the magnetopause near local noon, but skim the low-latitude flank magnetopause for long periods and there is therefore a higher probability of

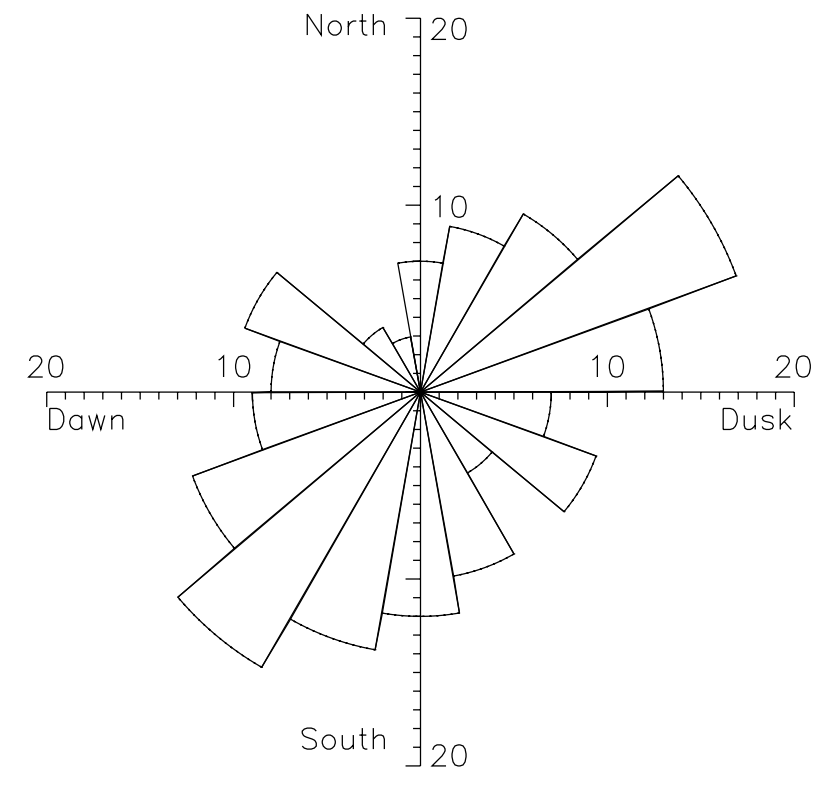

Fig. 2. A polar histogram of the IMF clock angle at the time of each magnetopause crossing. The figure takes the same format as Fig. 1.

observing at least one FTE on a low-latitude flank magnetopause crossing. Consequently, there are more observations of FTEs during $B_{Y}$-dominated IMF intervals than when $B_{Y}$ is low. A significant number of events (which were the subject of more detailed analysis by Fear et al., 2005b) were observed when the IMF was northward and dawnward.

Figure 2 shows a histogram of the IMF clock angle at the time of each of the 180 magnetopause crossings for which solar wind data were available. There was a tendency for the IMF to be either southward and dawnward, or northward and duskward (see Fig. 3 of Wang et al., 2006). Therefore a normalised distribution is shown in Fig. 3, using the following method which was adopted by Kuo et al. (1995). For those magnetopause passes on which FTEs were observed, the lagged IMF at the time of each FTE was taken, and averaged for each pass. For those passes on which no FTEs were observed, the IMF was taken to be that at the magnetopause crossing time. The number of passes on which FTEs were observed in each clock angle bin was then divided by the total number of passes to obtain an "FTE occurrence probability". For the purposes of this figure, the number of FTEs observed on a crossing is irrelevant; it shows the probability of one or more FTEs being observed. In the normalised distribution, FTEs are still most likely to be observed during IMF $B_{Y}$-dominated conditions, but there is no other clear peak in the distribution. The FTEs observed when the IMF was strongly northward were overwhelmingly observed on the post-terminator magnetopause (Fear et al., 2005b). 


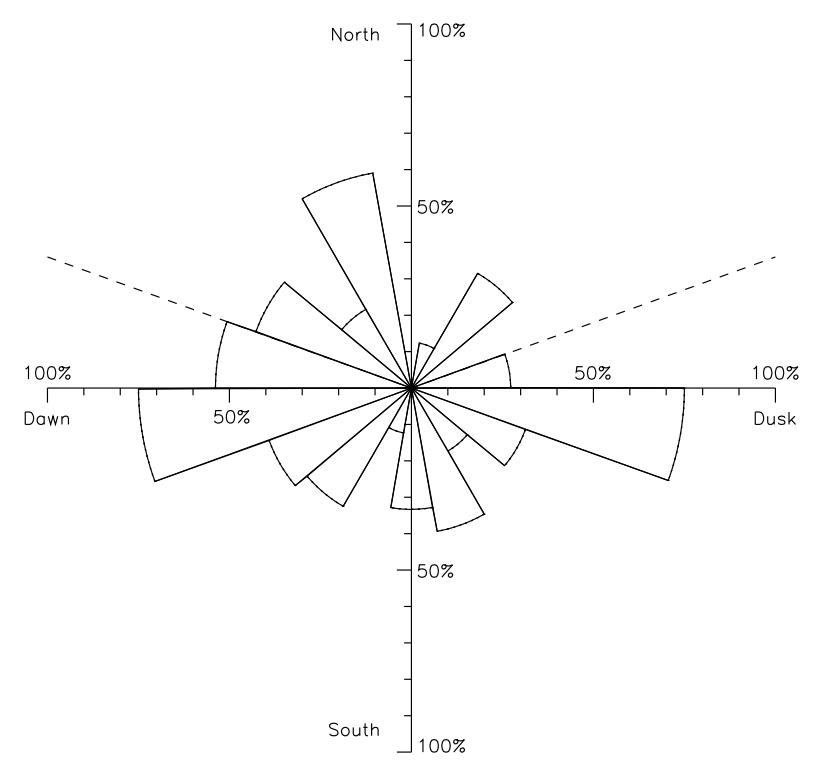

Fig. 3. The FTE "occurrence probability" (as defined by Kuo et al., 1995) for the survey carried out by Fear et al. (2005b) as a function of clock angle. The dashed line marks a clock angle of $\pm 70^{\circ}$.

\section{Multi-spacecraft timing analysis}

In order to make a case-by-case comparison with the Cooling et al. (2001) model, the technique described by Harvey $(1998, \mathrm{p} 311)$ was used to determine the velocity of the 213 FTEs which were observed with a bipolar $B_{N}$ signature on all four spacecraft. This technique uses the relative time differences between the signature observations at each of the six pairs of Cluster spacecraft, rather than the time differences between one "reference" spacecraft and the three others. This reduces the effect of an error on a single timing measurement on the outcome. It is also easily extendable to provide an estimate of the uncertainty on each calculated velocity.

An assumption made in multi-spacecraft timing analysis is that the structure being observed is planar on the scale of the spacecraft separation. In the case of an FTE, the timing analysis is not carried out on the surface of the FTE (which is likely to be curved on the scale of the $5000 \mathrm{~km}$ spacecraft separation). Instead, it is carried out on a plane that is constructed from the mid-points of the signature. This is illustrated in Fig. 4, which shows a sketch of an FTE, either in flux tube form (e.g. Russell and Elphic, 1978; Lee and $\mathrm{Fu}, 1985$, represented by solid circular/helical magnetic field lines), or in the form of a magnetopause "bulge" (Southwood et al., 1988; Scholer, 1988, represented by the dotted line). In this simple picture, the FTE is assumed to be symmetric. It is further assumed that the FTE does not change size or shape between being observed by the different spacecraft. The two bipolar traces represent the $B_{N}$ signatures observed by two

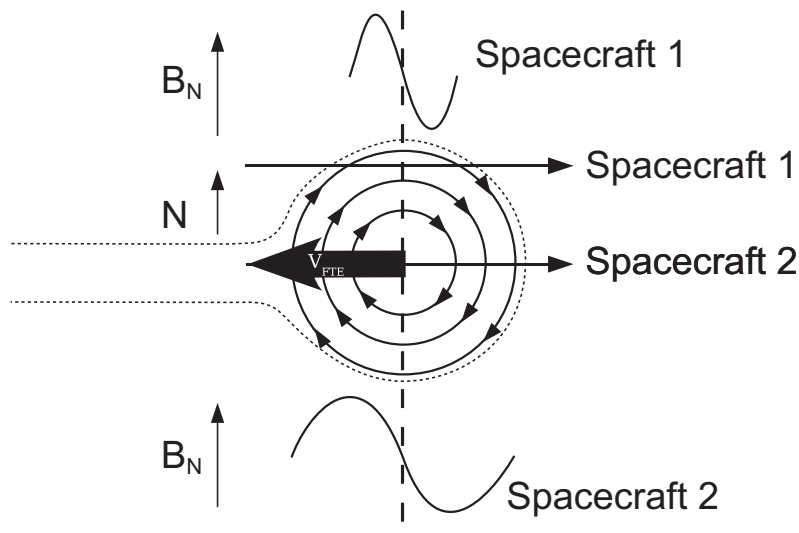

Fig. 4. An illustration of the $B_{N}$ signature observed by two spacecraft intersecting an FTE at different positions. An assumption made in the multi-spacecraft timing analysis is that the delay between the observed signatures at each spacecraft is due to the passage of a planar surface. In this figure, spacecraft 1 observes a briefer signature than spacecraft 2 , but the planar surface perpendicular to the direction of motion is that which passes through the mid-points of the $B_{N}$ signature (central dashed line). The plane is independent of any model of FTE structure. In this figure, a Russell and Elphic (1978) flux tube is indicated by solid circular field lines, whereas a Southwood et al. (1988) model FTE is indicated by the dotted line.

spacecraft which cross the FTE at different distances from the magnetopause (and indeed may only observe the magnetic field draping region around the FTE core). A spacecraft which enters deeper into the FTE should observe a longer duration $B_{N}$ signature (and may observe larger peak $B_{N}$ deflections) than a spacecraft which merely grazes the FTE. However, the mid-points of the $B_{N}$ signature observed by the different spacecraft (where $B_{N}$ is equal to its background value outside the FTE) form a plane which is perpendicular to the magnetopause and moves with the FTE.

Since there are four Cluster spacecraft, there are six possible time delays:

$t_{\alpha \beta}=t_{\beta}-t_{\alpha}$

where $t_{\alpha}$ and $t_{\beta}$ are the observation times at Cluster spacecraft $\alpha$ and $\beta(2 \leq \alpha \leq 4$, and $1 \leq \beta<\alpha)$. The magnetopause normal vector was determined by the Roelof and Sibeck (1993) model, as this was used to identify the events by Fear et al. (2005b), although we note that the bipolar $B_{N}$ signature is not sensitively dependent on the direction of the normal (Rijnbeek et al., 1984). The Roelof and Sibeck (1993) model takes the solar wind dynamic pressure $\left(P_{\text {dyn }}\right)$ and IMF $B_{Z}$ as inputs, but these inputs were capped if they were outside the model bounds $\left(-7 \mathrm{nT}<B_{Z}<7 \mathrm{nT}, P_{\text {dyn }}<8 \mathrm{nPa}\right.$ ). Any offset in the $B_{N}$ component was removed, and then each time delay $t_{\alpha \beta}$ was initially obtained by maximising the cross-correlation coefficient between the $B_{N}$ signatures at the 


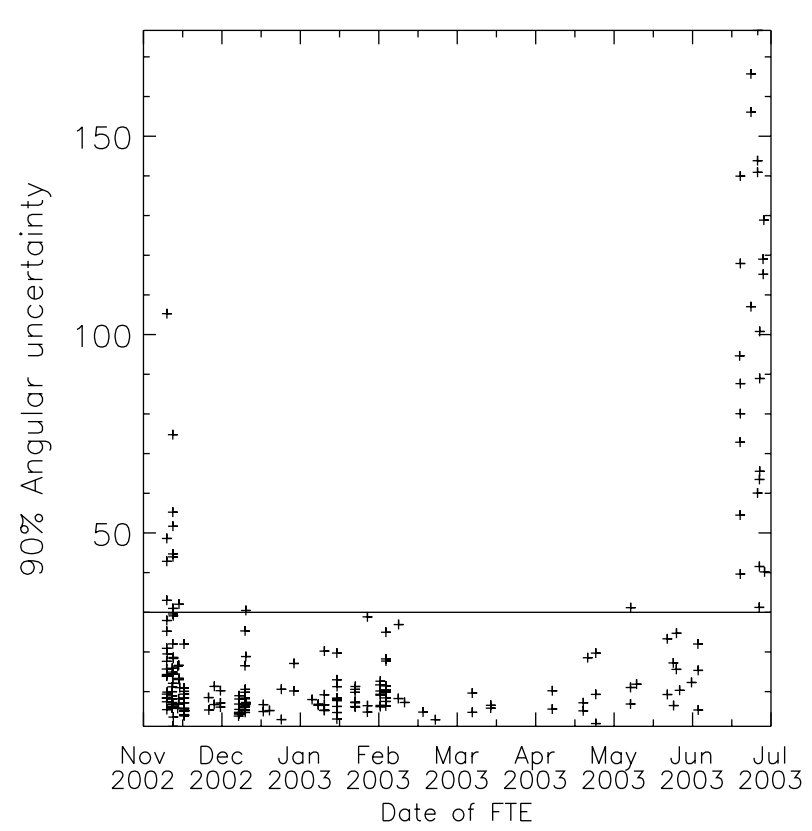

Fig. 5. The 90\% "angular uncertainty" error angle for each of the 213 FTEs for which a velocity was obtained. The line indicates the threshold of $30^{\circ}$ which was used to select well-defined events. At the beginning and end of the magnetopause crossing season the Cluster spacecraft tetrahedron quality was poor when the spacecraft crossed the magnetopause, and consequently some FTE velocities were determined with large errors. This was exacerbated in June by a spacecraft manoeuvre on 10 June 2003. All events after 10 June 2003 were discarded by Fear et al. (2005b), and are eliminated in the present study by applying a $30^{\circ}$ threshold on the $90 \%$ angular uncertainty.

two spacecraft. A preliminary examination of the $t_{\alpha \beta}$ values showed that sometimes the cross-correlation was dominated by part of the signature, such as the peak or trough of $B_{N}$, or some other internal structure that was present in the signature. Therefore $t_{\alpha \beta}$ was adjusted, where necessary, by eye to produce the best overall fit to three key features of the $B_{N}$ signatures on each spacecraft: the mid point of the bipolar signature (where $B_{N}$ is equal to the value outside the signature), and the positive and negative peaks. In order to assist in judging this correction, a low-pass filter was applied to produce a simplified signature which was examined alongside the unfiltered data. The cutoff period used in the filter was varied according to the duration of the FTE and any internal structure.

An uncertainty, or error, was also determined for each timing measurement $\left(\delta t_{\alpha \beta}\right)$, which was estimated such that all three of these features would match up within the uncertainty. In practice, many signatures differed slightly between two or more spacecraft, and so determining the time difference was not straightforward. In these cases, the following considerations were made:
1. If the duration of the $B_{N}$ signature observed by one spacecraft was shorter than the signature observed by the other, but the peaks were roughly symmetrical about the mid point, then the mid points were aligned and the peaks were not. The uncertainty on the time lag was estimated such that $t_{\alpha \beta} \pm \delta t_{\alpha \beta}$ encompassed the peaks.

2. If the $B_{N}$ signature was not symmetrical about the mid point on one or both spacecraft, then the peaks were aligned and the uncertainty was taken to be the difference in time which would be required to align the mid points of the signatures.

Following Harvey (1998, p. 311), the direction of motion of the FTE $(\hat{\boldsymbol{v}})$ and its speed $(V)$ were determined by minimising the function:

$S=\sum_{\alpha=1}^{4} \sum_{\beta=1}^{4}\left[\hat{\boldsymbol{v}} \cdot\left(\boldsymbol{r}_{\alpha}-\boldsymbol{r}_{\beta}\right)-V t_{\alpha \beta}\right]^{2}$

(Note that we use the vector $\hat{\boldsymbol{v}}$ instead of Harvey's $\hat{\boldsymbol{n}}$ in order to avoid confusion with the magnetopause normal.)

In Eq. (2), $\boldsymbol{r}_{\alpha}$ and $\boldsymbol{r}_{\beta}$ are the the position vectors of spacecraft $\alpha$ and $\beta$ relative to the tetrahedron mesocentre (Harvey, 1998, p310). The mesocentre is defined such that:

$$
\sum_{\alpha=1}^{4} \boldsymbol{r}_{\alpha}=\mathbf{0}
$$

Harvey (1998) defined the vector $\boldsymbol{m}$ as a vector with the direction of the FTE velocity but the magnitude of the reciprocal of the speed $\left(\boldsymbol{m}=\frac{\hat{v}}{V}\right)$. Consequently, the value of $\boldsymbol{m}$ for the minimum value of $S$ is given by:

$m_{l}=\frac{1}{16}\left[\sum_{\alpha \neq \beta} t_{\alpha \beta}\left(r_{\alpha k}-r_{\beta k}\right)\right] R_{k l}^{-1}$

where the tensor $R_{k l}$ is given by:

$R_{k l}=\frac{1}{4} \sum_{\alpha=1}^{4} r_{\alpha k} r_{\alpha l}$

and $r_{\alpha k}$ is the $k$ th component of the position vector of spacecraft $\alpha$ relative to the mesocentre.

This expression for $\boldsymbol{m}$ allows the velocity of an FTE to be calculated, assuming no uncertainty on the measurement of $t_{\alpha \beta}$. To estimate the uncertainty in the FTE velocity, we incorporated the estimated uncertainty in each of the individual spacecraft timings. We recalculated $\boldsymbol{m}$ using 10 time delays normally distributed about each $t_{\alpha \beta}$ measurement, with a standard deviation of $\delta t_{\alpha \beta} / 3$ (so $\boldsymbol{m}$ was recalculated $10^{6}$ times). If our measurement was robust, all values of $\boldsymbol{m}$ would cluster around the original value, otherwise the measurements were treated as ambiguous. We defined the angular uncertainty as the angle of a cone which contained $90 \%$ of the recalculated velocity vectors, and discarded events with an angular uncertainty of greater than $30^{\circ}$. 
The results of this error analysis are shown in Fig. 5. Throughout most of the magnetopause crossing season, the angular uncertainty on the FTE velocities was comparatively low. Even if there was a large uncertainty on the time delays between signatures observed at different spacecraft, the Cluster tetrahedron was of a good enough quality and the spacecraft separation was large enough that changing the time delays within the error bounds had very little effect on the resulting velocity. However, in November the Cluster spacecraft tetrahedron quality was poorer as the spacecraft crossed the magnetopause. Some FTEs exhibited very clear and similar signatures on all four spacecraft, resulting in well-defined velocities. However, the FTEs which exhibited weaker signatures at some or all of the spacecraft produced less welldefined velocities. On 10 June, a series of spacecraft manoeuvres rearranged the Cluster quartet into two pairs, drastically reducing the accuracy of the multi-spacecraft timing analysis. The events after this date were excluded by Fear et al. (2005b); all of them have an angular uncertainty greater than $30^{\circ}$.

Figure 6a shows a histogram of the peak-to-peak or "characteristic" time for each FTE observed by all four spacecraft ( $t_{\text {char }}$, as defined by Kawano et al., 1992). Where different spacecraft observed signatures of different durations for the same FTE, the most representative signatures were used. There is a wide range of values, but note that all but two FTEs have $t_{\text {char }}<90 \mathrm{~s}$, which corresponds to the category of transient magnetopause events exhibiting bipolar $B_{N}$ signatures that Kawano et al. (1992) ascribed to reconnection (as opposed to pressure pulse related events, which the authors concluded tended to have $t_{\text {char }}>90 \mathrm{~s}$ ).

In Fig. 6b, a histogram shows the angle between each $\boldsymbol{V}_{\text {FTE }}$ and the local model magnetopause surface (using the paraboloid model magnetopause surface used in the Cooling et al. (2001) model, which will be discussed in the next section). Events with an angular uncertainty of larger than $30^{\circ}$ have been excluded from this histogram. Most of the velocities lie close to the magnetopause surface, as would be expected as the structure moves along the magnetopause. However, 33 of the remaining events appeared to be directed away from the magnetopause surface by more than $30^{\circ}$ (the maximum angle being $78^{\circ}$ ).

Figure $6 \mathrm{c}$ shows the spread of FTE speeds. In both panels (c) and (d), we have excluded the events with a velocity directed out of the magnetopause surface by more than $30^{\circ}$, as well as the events with an angular uncertainty greater than $30^{\circ}$. This leaves 142 FTEs. Again, there is a large spread of speeds, with most events having $\sim 150<\left|\boldsymbol{V}_{\text {FTE }}\right|<\sim 550 \mathrm{~km} \mathrm{~s}^{-1}$.

By multiplying the characteristic time of an event by its speed, we can obtain a characteristic size of the FTE, which is shown in Fig. 6d. This characteristic size represents the scale length of the event along the direction of its motion across the spacecraft, although it is not exactly equal to the diameter of an FTE as the positive and negative peaks of the
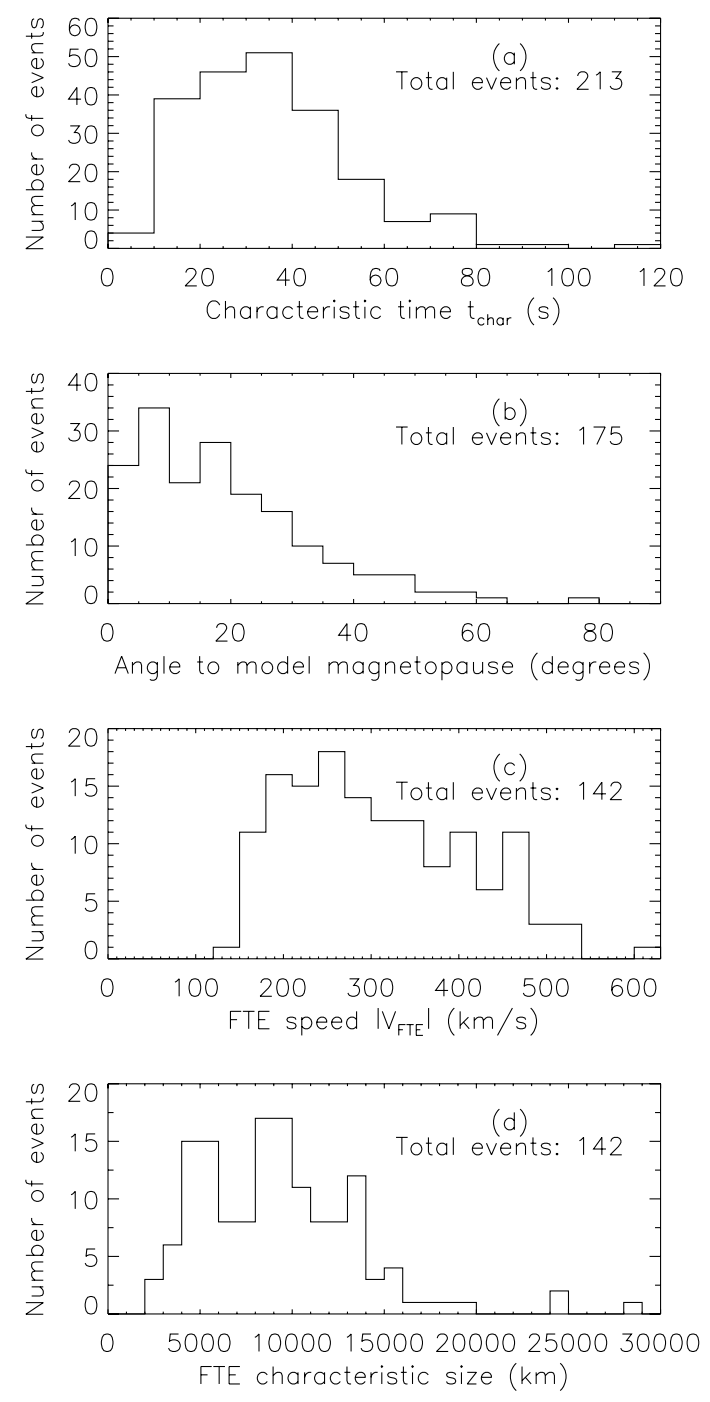

Fig. 6. Histograms of various properties of the FTEs observed by all four spacecraft. (a) The characteristic time $\left(t_{\text {char }}\right)$ for each of the FTEs observed by all four spacecraft. (b) The angle between the model FTE velocity $\boldsymbol{V}_{\text {FTE }}$ and the Cooling model magnetopause surface, for each of the FTEs observed by all four spacecraft and where the angular uncertainty on $V_{\text {FTE }}$ was less than $30^{\circ}$. (c) The speed of each FTE which had a velocity deduced to an angular uncertainty of less than $30^{\circ}$, and where the FTE velocity was within $30^{\circ}$ of the Cooling model magnetopause surface. (d) The characteristic size $\left(\left|\boldsymbol{V}_{\text {FTE }}\right| \times t_{\text {char }}\right)$ for each of the FTEs in (c).

bipolar $B_{N}$ signature do not necessarily represent the edges of the FTE core. (A bipolar signature is still observed if the spacecraft only sample the region of field line draping and do not enter the FTE core.) Nonetheless, this estimate provides the order of magnitude of the events. Most events have a characteristic size of between 4000 and $16000 \mathrm{~km}$ (0.6 to $2.5 R_{E}$ ), but events up to $29000 \mathrm{~km}\left(4.4 R_{E}\right)$ are observed moving along the magnetopause. Some events were 
observed that were smaller than $4000 \mathrm{~km}$, but these events will be underestimated as the FTE must be observed by all four Cluster spacecraft for its velocity to be determined, and the spacecraft had a typical separation scale of $5000 \mathrm{~km}$ during this season. Therefore, there may be many smaller-scale FTEs which are not included in this histogram. Of the events which are directed out of the magnetopause by more than $30^{\circ}$ (but with an angular uncertainty of less than $30^{\circ}$ ), one has a characteristic scale of $9 R_{E}$ (not shown), but the remainder are smaller than $29000 \mathrm{~km}$.

\section{The Cooling model}

The Cooling model calculates the motion of reconnected flux tubes over the surface of a model magnetopause for specified magnetosheath and solar wind conditions. It has been used by Wild et al. (2005), Fear et al. (2005b) and Dunlop et al. (2005) to explain the motion of flux transfer events. In this section, we explain the basic workings of the model.

Specifically, the Cooling model provides the velocity of the point at which reconnected magnetic flux threads the magnetopause. This instantaneous velocity is the velocity of the de Hoffmann-Teller frame $\left(\boldsymbol{V}_{H T}\right)$, which is the frame in which the electric field transforms to zero (de Hoffmann and Teller, 1950). This velocity may not always be the same as the velocity of an FTE calculated from multi-spacecraft timing analysis for two reasons. First, the velocity derived from a timing analysis is the velocity of the FTE perpendicular to the flux rope (or the equivalent structure in other models, e.g. Southwood et al., 1988; Scholer, 1988); the FTE axis is assumed to extend infinitely, so motion along the FTE axis cannot be determined. Second, the motion of part of an FTE further from the point at which it threads the magnetopause may be influenced more by local magnetosheath flows. However, we seek to evaluate how well this velocity represents the motion of observed FTEs.

Cowley and Owen (1989) derived the following simple relationships between the de Hoffmann-Teller velocities and the magnetosheath velocity, magnetic field and Alfvén speed from stress balance considerations:

$$
\begin{aligned}
& \boldsymbol{V}_{\mathrm{HTN}}=\boldsymbol{V}_{S H}-V_{A} \hat{\boldsymbol{b}}_{S H} \\
& \boldsymbol{V}_{\mathrm{HTS}}=\boldsymbol{V}_{S H}+V_{A} \hat{\boldsymbol{b}}_{S H}
\end{aligned}
$$

$\boldsymbol{V}_{\text {HTN }}$ and $\boldsymbol{V}_{\text {HTS }}$ are the de Hoffmann-Teller velocities of the flux tubes connected to the Northern and Southern Hemispheres respectively. $V_{S H}$ is the magnetosheath velocity, $V_{A}$ is the magnetosheath Alfvén speed, and $\hat{\boldsymbol{b}}_{S H}$ is the unit vector of the magnetosheath magnetic field at the point where the reconnected field lines cross the magnetopause.

In deriving these equations, Cowley and Owen (1989) assumed that the plasma flow along the reconnected flux tube is purely inward across the magnetopause (neglecting outward flow of magnetospheric plasma, reflection of magnetosheath plasma at the kink in the reconnected field line and mirroring of magnetosheath plasma at lower altitudes). They also assumed that, whilst the velocity of the plasma is changed as it crosses the magnetopause, its thermodynamic properties are unaffected and the plasma pressure just inside the magnetopause is the same as that in the magnetosheath. This region is referred to as the boundary layer. The boundary layer magnetic field is assumed to have the same direction as the local magnetospheric field, but to maintain the stress balance normal to the magnetopause the boundary layer magnetic pressure is the same as that in the magnetosheath. Therefore the magnetic field strengths in the boundary layer and magnetosheath are the same, and hence Alfvén speeds in the magnetosheath and boundary layer are also equal. Consequently, the boundary layer magnetic field strength is suppressed compared with the magnetospheric field.

The distinction between $\boldsymbol{V}_{\mathrm{HTN}}$ and $\boldsymbol{V}_{\mathrm{HTS}}$ arises from the plasma flow crossing the magnetopause, which is parallel or antiparallel to the magnetic field respectively. Therefore, these vectors will be unaffected if reconnection takes place with open geomagnetic field lines in the lobe, even though one of the resulting flux tubes is not connected to the ionosphere.

The Cooling model calculates the magnetosheath magnetic field $\left(\boldsymbol{B}_{m s}\right)$ from a model developed by Kobel and Flückiger (1994). The Kobel and Flückiger (1994) model takes three inputs: the stand-off distances of the bow shock and magnetopause $\left(R_{b s}\right.$ and $\left.R_{m p}\right)$ and the IMF. The magnetopause is modelled as a paraboloid. The magnetosheath velocity $\left(\boldsymbol{V}_{s h}\right)$ and density $\left(n_{s h}\right)$ are taken from an implementation of the Spreiter et al. (1966) model.

The magnetic field in the boundary layer just inside the magnetopause is assumed to have the magnitude of the model magnetosheath magnetic field, but the direction of the magnetospheric (geomagnetic) field $\left(\boldsymbol{B}_{g m}\right)$. This is a consequence of the simplifying assumption by Cowley and Owen (1989) that the discontinuity is purely rotational; therefore that all magnetosheath plasma incident upon the open magnetopause is transmitted across it into the boundary layer and that only the velocity of the plasma is changed (not its thermodynamic properties). The boundary layer and geomagnetic fields do not feature in the expressions for the velocities of reconnected field lines (Eqs. 6 and 7), but the geomagnetic field is required to evaluate the magnetic shear at the magnetopause and to trace the model reconnection line. The geomagnetic field direction is derived from a simple model where all geomagnetic field lines are mapped from the southern to the northern cusp over the surface of the magnetopause. The cusps are taken to be two points on the paraboloid magnetopause at the locations $\left(\frac{1}{2} R_{m p}, 0, \pm R_{m p}\right)_{\mathrm{GSM}}$.

In the original implementation of the Cooling model, a reconnection site is specified by the user. The model permits reconnection at this site if the difference in the components of the magnetic field perpendicular to the magnetopause current 
direction (i.e. $\left|\boldsymbol{B}_{m s}-\boldsymbol{B}_{g m}\right|$ ) is above a user-defined threshold. A component reconnection line is then formed by tracing along the direction of the magnetopause current, i.e. perpendicular to $\left(\boldsymbol{B}_{m s}-\boldsymbol{B}_{g m}\right)$. Model flux tubes are placed at several positions along the reconnection line; Eqs. (6) and (7) are evaluated at those points and the flux tubes are moved a step of $V_{\text {HTN }} \Delta T$ or $V_{\text {HTS }} \Delta T$, where $\Delta T$ is a short time interval. This process is repeated, and so the paths of the model reconnected flux tubes are traced. However, all that is needed to calculate the instantaneous velocity of an FTE using the expressions derived by Cowley and Owen (1989) are the local magnetosheath parameters $\left(\boldsymbol{B}_{m s}, \boldsymbol{V}_{s h}\right.$ and $\left.n_{s h}\right)$. Therefore in this study, the model is run in reverse: Eqs. (6) and (7) are evaluated at the location of an observed FTE (projected onto the model magnetopause used by the Cooling model), and the observed FTE velocity is compared with the two model velocities ( $\boldsymbol{V}_{\mathrm{HTN}}$ and $\boldsymbol{V}_{\mathrm{HTS}}$ ) at this point. A step is taken in the opposite direction for each model flux tube ( $-V_{H T} \Delta T$, where $\Delta T$ is $0.75 \mathrm{~s}$ ) and the model flux tube velocity is reevaluated. This process is repeated for 1000 steps (or $750 \mathrm{~s}$ ).

\section{Case-by-case comparison}

A separate model run was carried out for each of the 213 FTEs observed by all four spacecraft using the lagged IMF, solar wind velocity and solar wind density for each event. An example model run is shown in Fig. 7, which shows a case where the observed FTE velocity (green arrow) matches well with the Cooling model $\boldsymbol{V}_{\mathrm{HTN}}$ (black arrow at the end of the red line), but not the model $\boldsymbol{V}_{\text {HTS }}$ (black arrow at the end of the blue line). The motion of the model reconnected field lines that form the FTE can be traced back along the red line towards a subsolar reconnection line which has been initiated at $Y_{\mathrm{GSM}}=Z_{\mathrm{GSM}}=0$. The precise reconnection site cannot be determined from the model alone - an FTE generated anywhere on the red path would have the same subsequent motion. The lagged IMF for this FTE was slightly northward, but predominantly duskward. The point at which the model magnetosheath flow becomes super-Alfvénic is marked in Fig. 7 by a purple contour.

The magnetopause stand-off distance $\left(R_{m p}\right)$, was calculated separately for each event:

$R_{m p}=\left(\frac{B_{E}^{2}}{\mu_{0} n_{s w} m_{i} v_{s w}^{2}}\right)^{\frac{1}{6}}$

(Schield, 1969), where $n_{s w}$ is the solar wind ion density and $v_{s w}$ is the solar wind speed, both of which were taken from the lagged ACE data, $B_{E}$ is the equatorial magnetic field strength at the Earth's surface (taken to be $3.1 \times 10^{4} \mathrm{nT}$ ), $\mu_{0}$ is the permeability of free space and $m_{i}$ is the proton mass. The presence of heavier ions in the solar wind was neglected. The

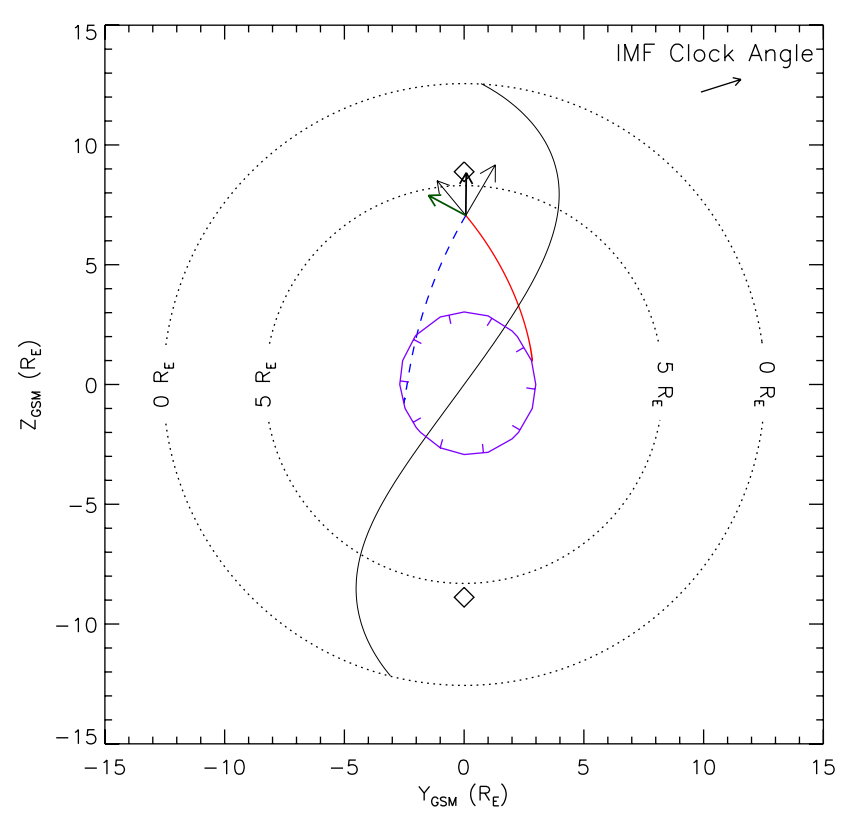

Fig. 7. An example model run: 22 February 2003, 01:23 UT. The figure shows a view of the model magnetopause projected into the GSM Y-Z plane, with concentric dotted circles marking contours of $\mathrm{X}_{\mathrm{GSM}}$; the cusps are considered to be point singularities marked by diamonds. The boundary at which the model magnetosheath flow becomes super-Alfvénic is marked by a purple contour, with tick marks pointing to the direction in which the flow is sub-Alfvénic. A model subsolar component reconnection line (thin black line) has been initiated at $\left(R_{m p}, 0,0\right)_{\mathrm{GSM}}$ and traced perpendicular to $\left(\boldsymbol{B}_{m s}-\boldsymbol{B}_{g m}\right)$ for $20 R_{E}$ in each direction. The position and velocity of the observed FTE have been projected onto the model magnetopause. The projected observed FTE velocity $\left(\boldsymbol{V}_{\text {projected }}\right)$ is shown as a green arrow. The model velocities for flux tubes connected to the Northern and Southern Hemispheres ( $\boldsymbol{V}_{\text {HTN }}$ and $\boldsymbol{V}_{\text {HTS }}$ ) have also been calculated using the model magnetosheath magnetic field and flow speed at the projected position (black arrows). The model flux tube paths have been traced backward for $750 \mathrm{~s}$ (red line: flux tubes connected to Northern Hemisphere; blue line: connection to Southern Hemisphere). Also shown as a black arrow is the model (radial) magnetosheath flow.

observed FTE velocity $\left(\boldsymbol{V}_{\mathrm{FTE}}\right)$ was projected onto the model magnetopause surface:

$$
\boldsymbol{V}_{\text {projected }}=\hat{\boldsymbol{n}}_{\text {model }} \times\left(\boldsymbol{V}_{\mathrm{FTE}} \times \hat{\boldsymbol{n}}_{\text {model }}\right)
$$

In the simple case of reconnection near the subsolar point, the reconnected field lines move in opposite directions. Consequently only FTEs connected to the Northern Hemisphere would be observed northward of a subsolar reconnection line, and only FTEs connected to the Southern Hemisphere would be observed southward (although the reconnection line will be tilted if the IMF has a significant $B_{Y}$ component). However, as noted by Daly et al. (1984) and Fear et al. (2005b), if reconnection occurs away from the subsolar region then reconnected flux tubes at the magnetopause may be connected 


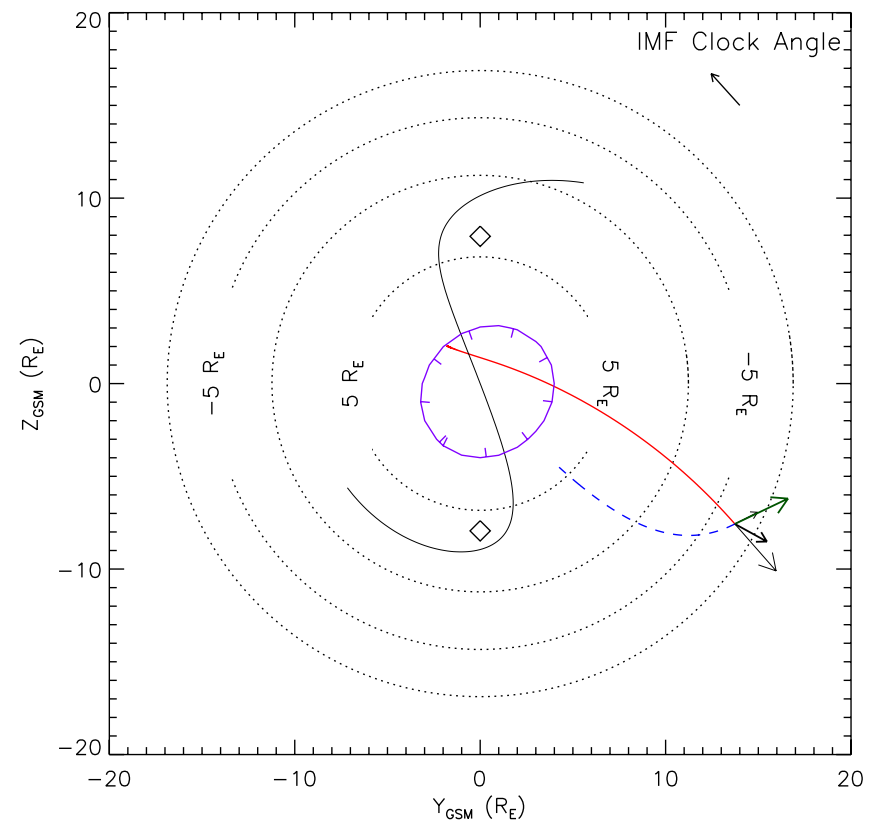

Fig. 8. An example model run for an FTE which is connected to the hemisphere opposite to that which would be inferred from the position relative to a subsolar component reconnection line (12 November 2002, 14:42 UT). This figure takes the same format as Fig. 7. The direction of motion of the FTE is consistent with the model $\boldsymbol{V}_{\text {HTS }}$. The equatorward motion of the FTE is therefore a consequence of super-Alfvénic magnetosheath flow at a high-latitude reconnection site (discussed by Fear et al., 2005b).

to the opposite hemisphere from that which would be expected in the near-subsolar scenario. Therefore the existence of two possible FTE velocities $\left(\boldsymbol{V}_{\mathrm{HTN}}\right.$ and $\left.\boldsymbol{V}_{\mathrm{HTS}}\right)$ at such a site is not merely an artefact of the model. An example is shown in Fig. 8, which shows the comparison with the model for an FTE observed on the 12 November 2002 at 14:42 UT. This FTE occurred during an interval of strongly northward IMF, and was part of a group of FTEs studied by Fear et al. (2005b). As the IMF is strongly northward, the model subsolar component reconnection line is highly tilted. Therefore, if strict subsolar reconnection was assumed, only reconnected field lines connected to the Northern Hemisphere would be observed at this point (as illustrated by the red line in Fig. 8), as field lines opened at a subsolar reconnection site and connected to the Southern Hemisphere would move dawnward. (A subsolar reconnection scenario, combined with re-reconnection, was one of the explanations for northward IMF FTEs proposed by Kawano and Russell, 1997a,b). However, as discussed by Fear et al. (2005b), this FTE exhibited equatorward motion as it was formed at a high-latitude reconnection site where the magnetosheath flow was superAlfvénic, and was connected to the Southern Hemisphere (as indicated by the similarity of the projected FTE velocity to the model $\boldsymbol{V}_{\text {HTS }}$ vector). Such an FTE could not be observed

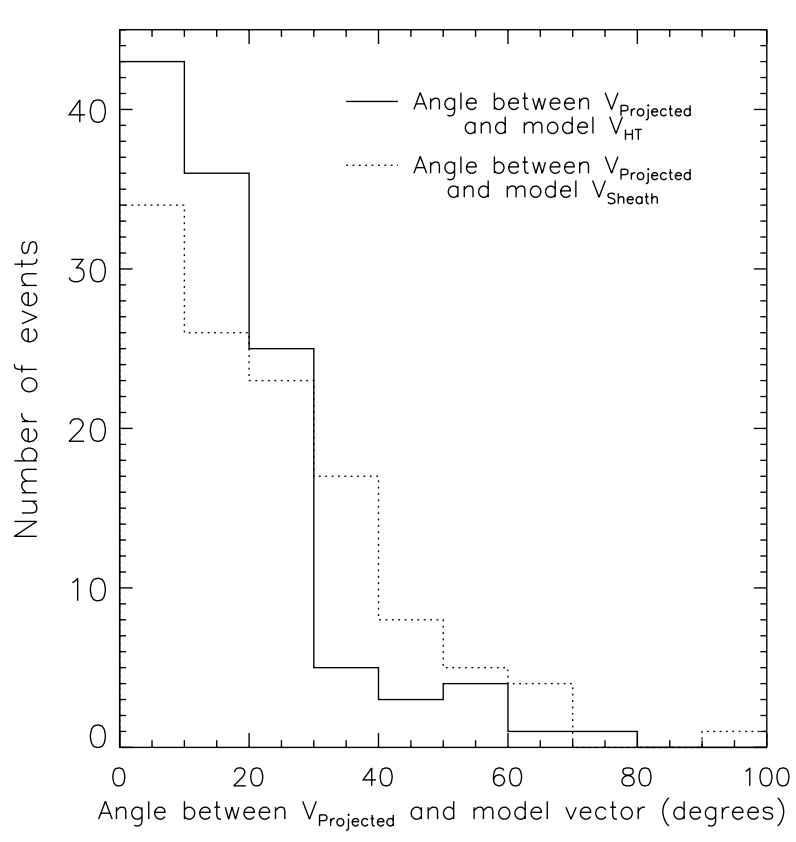

Fig. 9. Histograms of the angle between the observed FTE velocities (projected onto the model magnetopause) and (a) the nearest Cooling model velocity $\left(\boldsymbol{V}_{\mathrm{HTN}}\right.$ or $\left.\boldsymbol{V}_{\mathrm{HTS}}\right)$, and (b) the model magnetosheath velocity (dotted line). These histograms include all FTEs which had an angular uncertaintly of less than $30^{\circ}$, a velocity within $30^{\circ}$ of the model magnetopause surface and a characteristic scale of greater than $5000 \mathrm{~km}$ (total 118 events).

at this location if it had been generated in a region where the magnetosheath flow was sub-Alfvénic, as it would have moved dawnward under the effects of magnetic tension. As a result, the blue path in Fig. 8 cannot be traced back into the region of sub-Alfvénic flow.

Consequently, the projected velocity of each FTE was compared with both the model $\boldsymbol{V}_{\mathrm{HTN}}$ and $\boldsymbol{V}_{\mathrm{HTS}}$ vectors. The FTE velocity was regarded as consistent with $\boldsymbol{V}_{\mathrm{HTN}}$ or $\boldsymbol{V}_{\text {HTS }}$ if $\boldsymbol{V}_{\text {projected }}$ was within $30^{\circ}$ of the model velocity, and $\left|\boldsymbol{V}_{\text {projected }}\right|$ was greater than half and less than double the model speed. Out of the 142 events where $\boldsymbol{V}_{\text {FTE }}$ was determined with a $90 \%$ angular uncertainty of less than $30^{\circ}$, and where $\boldsymbol{V}_{\mathrm{FTE}}$ was within $30^{\circ}$ of the model magnetopause surface, 103 events were consistent with either the model $\boldsymbol{V}_{\mathrm{HTN}}$ or $\boldsymbol{V}_{\text {HTS }}$ in magnitude and direction (73\%). Interestingly, if we exclude the events with a characteristic size of less than $5000 \mathrm{~km}$, we find that 92 out of 118 events are consistent with either $\boldsymbol{V}_{\mathrm{HTN}}$ or $\boldsymbol{V}_{\mathrm{HTS}}$ in both magnitude and direction (78\%), and a further 12 events (a total of 88\%) are consistent with either $\boldsymbol{V}_{\text {HTN }}$ or $\boldsymbol{V}_{\text {HTS }}$ in direction only (such as the example in Fig. 8).

A comparison of the angle between the observed FTE velocities (projected onto the magnetopause surface) and the Cooling model velocities and the model (radial) 
magnetosheath velocity is shown in Fig. 9. In these histograms, events with an angular uncertainty of greater than $30^{\circ}$, a velocity out of the magnetopause surface by more than $30^{\circ}$ or a characteristic scale of less than $5000 \mathrm{~km}$ have been excluded. The solid line is a histogram of the angle between $\boldsymbol{V}_{\text {Projected }}$ and $\boldsymbol{V}_{H T}$ (which is taken to be the nearer of the two model FTE velocities). There is a strong peak in this distribution at angles below $30^{\circ}$. On the other hand, the histogram of the angle between $\boldsymbol{V}_{\text {Projected }}$ and the model magnetosheath velocity shows a broader distribution with a higher mean (dotted line). This indicates that the Cooling model explains FTE motion better than a simple assumption of radial motion away from the subsolar point.

\subsection{Evaluation of $\boldsymbol{V}_{H T}$ from magnetosheath parameters}

Obvious causes of error in the Cooling model comparison include the solar wind lag and the accuracy of the models used to calculate the magnetosheath density, flow velocity and magnetic field. When the FTE is observed by at least one spacecraft in the magnetosheath, these errors can be eliminated by calculating the de Hoffmann-Teller velocites given by Eqs. (6) and (7) using the observed magnetosheath parameters, before and after the passage of the FTE, rather than model values.

We have evaluated Eqs. (6) and (7) for the remaining 12 magnetosheath FTEs which had an angular uncertainty of less than $30^{\circ}$, a velocity within $30^{\circ}$ of the model magnetopause surface and a characteristic size of greater than $5000 \mathrm{~km}$ but which were not consistent in direction with the Cooling model. (Two further events were observed when all four spacecraft were in the magnetosphere.) For each FTE, we took typical magnetosheath conditions outside the FTE, and used the magnetic field observed by FGM and the ground-calibrated velocity and density moments derived from CIS HIA data. (The HIA sensor is less prone to saturation in the magnetosheath than CODIF, and therefore provides more reliable moments in the magnetosheath.) The mean difference between $\boldsymbol{V}_{H T}$ calculated with the Cooling model and using Eqs. (6) and (7) and the observed magnetosheath parameters was only $8^{\circ}$ in the case of model vectors for field lines that were connected to the hemisphere in which the FTE was observed (accounting for any $B_{Y}$-induced tilt of the subsolar reconnection line), but the mean difference was $29^{\circ}$ for the field lines which were connected to the opposite hemisphere. This indicates that the Cooling model results are reasonably stable when the magnetosheath flow and magnetic tension force act in broadly the same direction, but the model is much less stable if the flow is super-Alfvénic and the magnetic tension is oppositely directed (which results in the FTE being dragged back across the reconnection site). The mean angular difference between the observed and model magnetosheath flow velocity was $10^{\circ}$. Two of the 12 FTEs which were inconsistent with the Cooling model $\boldsymbol{V}_{H T}$ vectors were consistent with one of the vectors calculated from the magnetosheath parameters (both were consistent with $\boldsymbol{V}_{\text {HTS }}$, but were observed on the northward side of the subsolar reconnection line used in the model).

\section{Plasma signatures}

Examination of the plasma signatures of FTEs can remove the ambiguity of the two possible model velocities, $\boldsymbol{V}_{\text {HTN }}$ and $\boldsymbol{V}_{\text {HTS }}$. If a magnetosheath FTE is observed, and the spacecraft enters onto reconnected magnetic field lines (as opposed to observing only the region of magnetic field draping), then one expects to observe escaping magnetosphericenergy and energised magnetosheath-energy ions and electrons moving parallel to the magnetic field if the open magnetic field lines are connected to the Southern Hemisphere, or antiparallel to the magnetic field if the field lines are connected to the Northern Hemisphere. If a magnetospheric FTE is observed, then the magnetosheath population crossing into the magnetosphere may mirror at low altitudes and form a bidirectional field-aligned population. However, if a unidirectional magnetosheath population is observed, it will be aligned parallel to the magnetic field if the flux tube is connected to the Northern Hemisphere, and antiparallel to the magnetic field if connected to the Southern Hemisphere. There may also be a drop-out of the magnetospheric-energy ions and electrons.

To further test the Cooling model, and remove the ambiguity of two model velocities, we examine the plasma signatures of the FTEs in Sect. 5 which were consistent in direction with $\boldsymbol{V}_{\text {HTN }}$ or $\boldsymbol{V}_{\text {HTS }}$ (but not both, as may be the case if the magnetosheath flow dominates over the Alfvénic component of Eqs. 6 and 7). Unfortunately, due to the degradation of the microchannel plates in some of the PEACE instruments, PEACE was turned off on all of the spacecraft whilst in the magnetosheath for much of the season. We are therefore restricted to those events on a few crossings for which PEACE data are available on at least one spacecraft, mainly at the beginning and end of the season. CIS data were more generally available throughout the season, but we examine here only those events for which PEACE data were also available. We also briefly examine some of the highenergy signatures observed by the RAPID instrument.

The results are shown in Table 1, which lists all 27 FTEs which satisfied the conditions outlined above. 13 of these events occurred when the absolute IMF clock angle was less than $70^{\circ}$ and were observed on the 10,12 or 17 November 2002. These events are denoted with an asterisk and were studied by Fear et al. (2005b), although their plasma signatures were not examined in that paper and so are presented here. The first two columns list the event number (allocated to each event observed by all four spacecraft, and used to refer to FTEs in this paper) and the date and time of each FTE. The third column shows which of the two Cooling model de Hoffmann-Teller velocities fits the observed FTE velocity 
Table 1. A list of the plasma signatures observed for events which were consistent in angle with only one Cooling model velocity. "Epoch" refers to the time of observation of the FTE. "Model fit" shows which of the two Cooling model de Hoffmann-Teller velocities was consistent with the observed FTE velocity. "PEACE signature" and "CIS signature" shows whether a unidirectional electron or ion signature was observed by at least one spacecraft, and whether the signature is in pitch angles parallel or antiparallel to the magnetic field. Bidirectional electron or ion signatures are also noted if no unidirectional signature was observed. It is noted whether the signature is consistent with the hemisphere of connection of the model de Hoffmann-Teller velocity from the Cooling model, bearing in mind whether the FTE was observed inside or outside the magnetopause. Where the Cluster tetrahedron straddled the magnetopause, the magnetosheath signatures are given in this table, as this reduces the ambiguity introduced in magnetospheric signatures by ions and electrons mirroring at low altitudes.

\begin{tabular}{|c|c|c|c|c|c|c|}
\hline FTE No. & Epoch & Model fit & PEACE signature & PEACE consistent? & CIS signature & CIS consistent? \\
\hline $1 *$ & 10 Nov 2002 10:18 & $\boldsymbol{V}_{\mathrm{HTN}}$ & Bidirectional & & Antiparallel & YES \\
\hline $8^{*}$ & 10 Nov 2002 11:05 & $\boldsymbol{V}_{\mathrm{HTN}}$ & Bidirectional & & Antiparallel & YES \\
\hline $10^{*}$ & 10 Nov 2002 11:19 & $V_{\mathrm{HTN}}$ & No signature & & No signature & \\
\hline $21 *$ & 12 Nov 2002 13:11 & $\boldsymbol{V}_{\mathrm{HTS}}$ & Parallel & YES & Antiparallel & NO \\
\hline $23 * \dagger$ & 12 Nov 2002 13:53 & $V_{\text {HTN }}$ & Parallel & YES & Parallel & YES \\
\hline $25 * \dagger$ & 12 Nov 2002 14:20 & $V_{\text {HTN }}$ & Parallel & YES & Parallel & YES \\
\hline $27 *$ & 12 Nov 2002 14:40 & $\boldsymbol{V}_{\mathrm{HTS}}$ & Parallel & YES & Antiparallel & NO \\
\hline $28 *$ & 12 Nov 2002 14:42 & $\boldsymbol{V}_{\mathrm{HTS}}$ & Parallel & YES & Antiparallel & $\mathrm{NO}$ \\
\hline $29 *$ & 12 Nov 2002 14:44 & $\boldsymbol{V}_{\mathrm{HTS}}$ & Parallel & YES & Antiparallel & $\mathrm{NO}$ \\
\hline 31 & 12 Nov 2002 16:27 & $V_{\text {HTN }}$ & Parallel & NO & Antiparallel & YES \\
\hline 49 & 14 Nov 2002 16:42 & $\boldsymbol{V}_{\mathrm{HTS}}$ & Bidirectional & & Bidirectional & \\
\hline $59 *$ & 17 Nov 2002 02:52 & $\boldsymbol{V}_{\text {HTS }}$ & Parallel & YES & Parallel & YES \\
\hline $61 \ddagger$ & 17 Nov 2002 03:24 & $\S \boldsymbol{V}_{\mathrm{HTN}}$ & Parallel & $\S \mathrm{NO}$ & Antiparallel & $\S Y E S$ \\
\hline $63 * \neq$ & 17 Nov 2002 04:47 & $\boldsymbol{V}_{\mathrm{HTS}}$ & Parallel & YES & Antiparallel & NO \\
\hline $64 *+$ & 17 Nov 2002 05:08 & $\boldsymbol{V}_{\mathrm{HTS}}$ & Parallel & YES & Antiparallel & NO \\
\hline $65^{* \ddagger}$ & 17 Nov 2002 05:09 & $\boldsymbol{V}_{\mathrm{HTS}}$ & Bidirectional & & Antiparallel & NO \\
\hline $108 \ddagger$ & 24 Dec 2002 15:50 & $V_{\text {HTN }}$ & Antiparallel & YES & Unclear & \\
\hline 161 & 22 Feb 2003 01:23 & $\boldsymbol{V}_{\text {HTN }}$ & Antiparallel & YES & Antiparallel & YES \\
\hline $163 \ddagger$ & 8 Mar 2003 07:07 & $\boldsymbol{V}_{\text {HTN }}$ & Bidirectional & & Parallel & NO \\
\hline 164 & 15 Mar 2003 09:55 & $V_{\text {HTN }}$ & Bidirectional & & Antiparallel & YES \\
\hline $167 \ddagger$ & 8 Apr 2003 03:57 & $V_{\text {HTN }}$ & Antiparallel & YES & Antiparallel & YES \\
\hline 178 & 22 May 2003 12:26 & $V_{\text {HTN }}$ & Parallel & NO & Parallel & NO \\
\hline 180 & 24 May 2003 19:57 & $\boldsymbol{V}_{\text {HTN }}$ & Parallel & $\mathrm{NO}$ & Parallel & NO \\
\hline 181 & 24 May 2003 22:25 & $\boldsymbol{V}_{\mathrm{HTN}}$ & Bidirectional & & Unclear & \\
\hline 182 & 25 May 2003 23:54 & $V_{\text {HTN }}$ & No signature & & Parallel & NO \\
\hline 183 & 26 May 2003 01:13 & $V_{\text {HTN }}$ & Bidirectional & & Unclear & \\
\hline 187 & 3 Jun 2003 09:57 & $\boldsymbol{V}_{\mathrm{HTS}}$ & Bidirectional & & No signature & \\
\hline
\end{tabular}

* Indicates an event examined by Fear et al. (2005b): IMF clock angle magnitude less than $70^{\circ}$ on the 10,12 or 17 November.

$\dagger$ Events only observed by spacecraft inside the magnetopause (in the magnetosphere-proper or a boundary layer).

$\ddagger$ Events which occurred when the Cluster spacecraft were in burst mode.

$\S$ FTE 61 is consistent with $\boldsymbol{V}_{\text {HTS }}$ and not with $\boldsymbol{V}_{\text {HTN }}$ if the model velocities are calculated from observed magnetosheath parameters. In the light of this calculation, the PEACE electron signature is consistent with this velocity, but the ion signature is not.

(projected onto the model magnetopause). The fourth and fifth columns summarise the PEACE observations, and the final two columns summarise the CIS observations. Magnetosheath plasma data were available from at least one Cluster spacecraft for all events, except for FTEs 23 and 25, which were only observed by spacecraft in a boundary layer on the Earthward side of the magnetopause.

\subsection{PEACE signatures}

The electron signature of a magnetosheath FTE has two parts, in addition to the undisturbed magnetosheath popula- tion which has yet to interact with the magnetopause. These are the escaping magnetospheric population, and an accelerated magnetosheath population which has either been reflected by the "kink" of the reconnected field line at the magnetopause, or been transmitted across the magnetopause, mirrored at low altitudes and crossed the magnetopause a second time. Examination of the events listed in Table 1 reveals that the accelerated magnetosheath population is more commonly observed by the PEACE instruments.

The PEACE pitch angle distributions (PADs) were rebinned to pitch angles on the ground, as this improves their reliability. Very few events exhibited a clear electron 

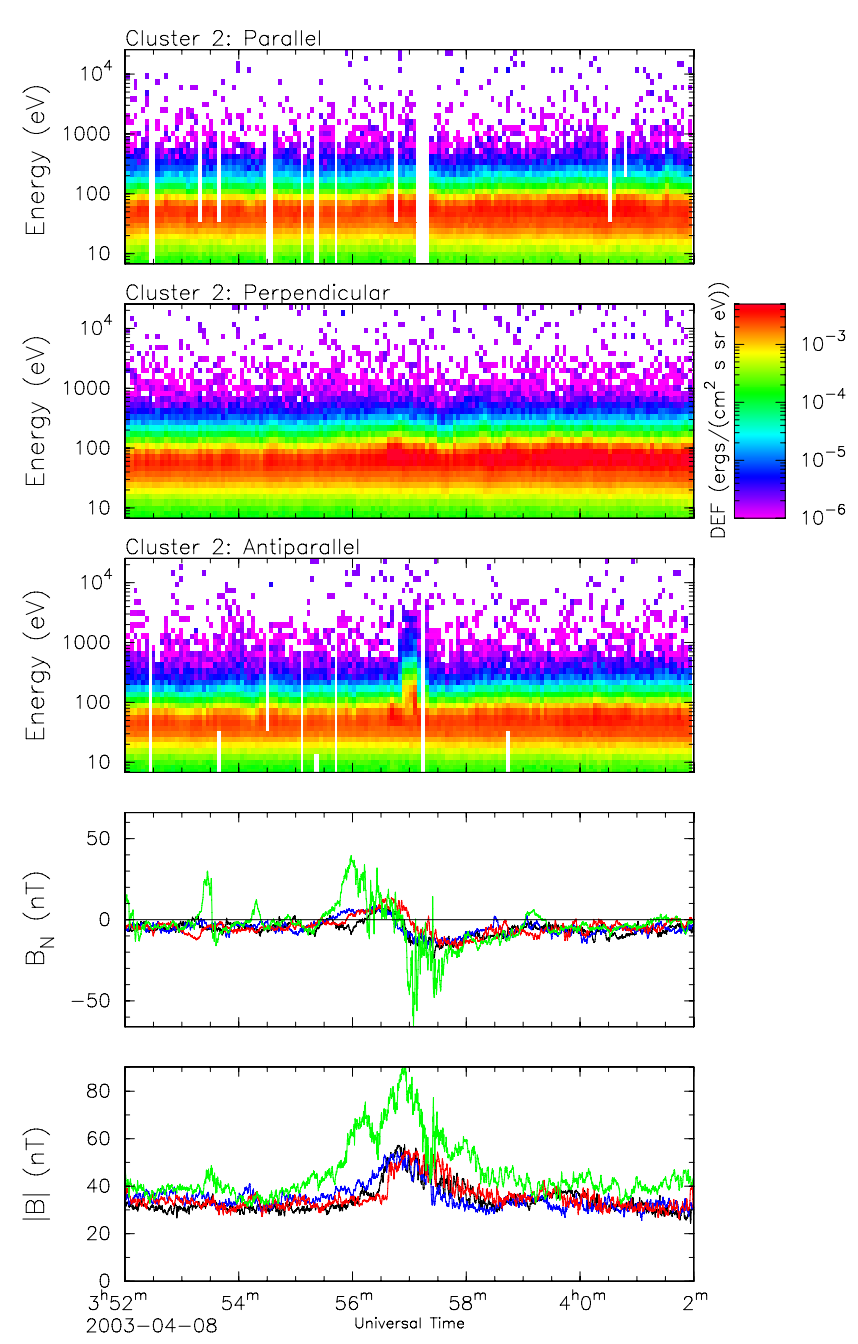

signature on at least one spacecraft, localised to the observed FTE, in pitch angles either parallel to or antiparallel to the magnetic field, without any form of signature in the opposite pitch angle. One event which did was FTE 167 (8 April 2003, 03:57 UT), which is shown in Fig. 10a. The figure shows the electron and FGM data from Cluster 2 for FTE 167. The isotropic, low energy electron plasma $(10-100 \mathrm{eV})$ observed in the top three panels demonstrates that the spacecraft was in the magnetosheath. At 03:57 UT, there was a brief signature of energised magnetosheath electrons moving antiparallel to the magnetic field, which is consistent with a connection to the Northern Hemisphere. The FTE was observed northward of a tilted subsolar reconnection line, and the observed FTE velocity was consistent with the model $\boldsymbol{V}_{\text {HTN }}$ (shown in Fig. 10b). A similar signature was observed by Cluster 4 . A more bidirectional electron signature was observed by Cluster 3 which was the nearest spacecraft to the magnetopause, and which observed much more structure in the $B_{N}$ signature

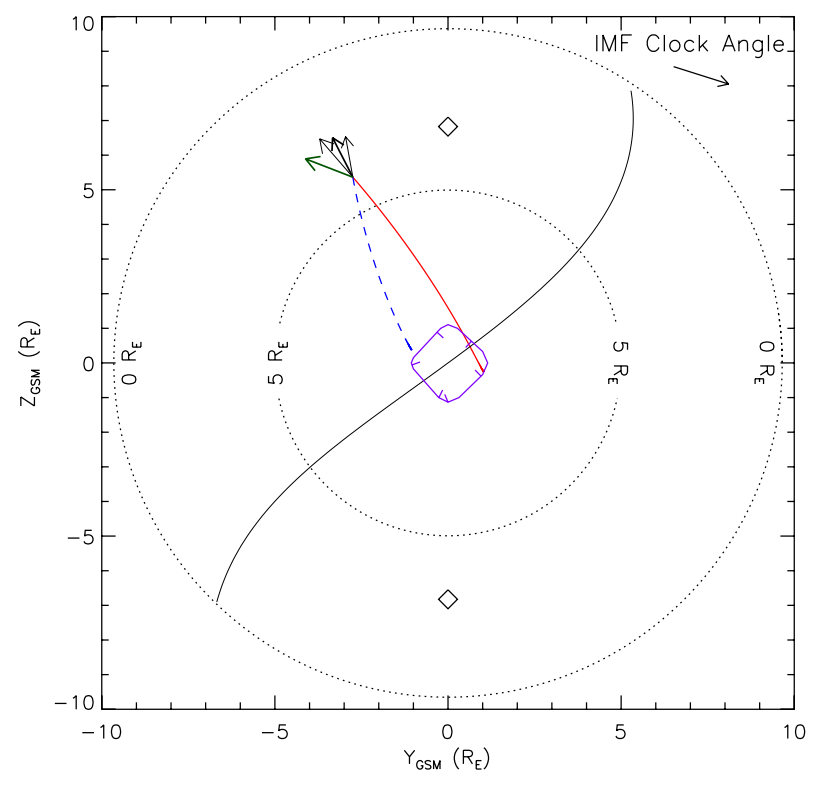

Fig. 10. (a: left) PEACE electron data from Cluster 2 for FTE 167 (8 April 2003, 03:57 UT). The top three panels are spectrograms showing the differential energy flux of electrons moving parallel, perpendicular and antiparallel to the magnetic field respectively. The bottom two panels show the normal component of the magnetic field and the magnetic field strength observed by all four spacecraft in standard Cluster colours (C1: black, C2: red, C3: green, C4: blue). This event is an example of a straightforward electron signature (accelerated magnetosheath plasma antiparallel to the magnetic field, indicating a connection to the Northern Hemisphere). (b: above) The Cooling model run for this event, in the same format as Fig. 7. The observed velocity was consistent with the model de HoffmannTeller velocity for a flux tube connected to the Northern Hemisphere. This is consistent with the antiparallel electron signature observed by PEACE.

(Fig. 10a, bottom two panels). FTEs 49, 108 and 180 exhibited similar signatures (although not necessarily as strong) on at least one spacecraft, with the directionality indicated in Table 1.

The remaining events which did exhibit a clear directionality also exhibited a weaker accelerated electron signature in the opposite direction, although this was usually not localised to the magnetic field signature of the FTE. The electron signatures for FTE 161 (22 February 2003, 01:23 UT) are shown in Fig. 11 (the Cooling model run for this event was shown in Fig. 7). The data shown are from Clusters 1 and 2. Throughout the interval, the magnetosheath electron plasma observed by Cluster 1 was anisotropic, as the electron distribution observed parallel to the magnetic field extended to higher energies than those observed antiparallel to the magnetic field. However, at 01:23 UT, when the magnetic field signature of the FTE was observed, there was a burst of accelerated magnetosheath electrons antiparallel to 

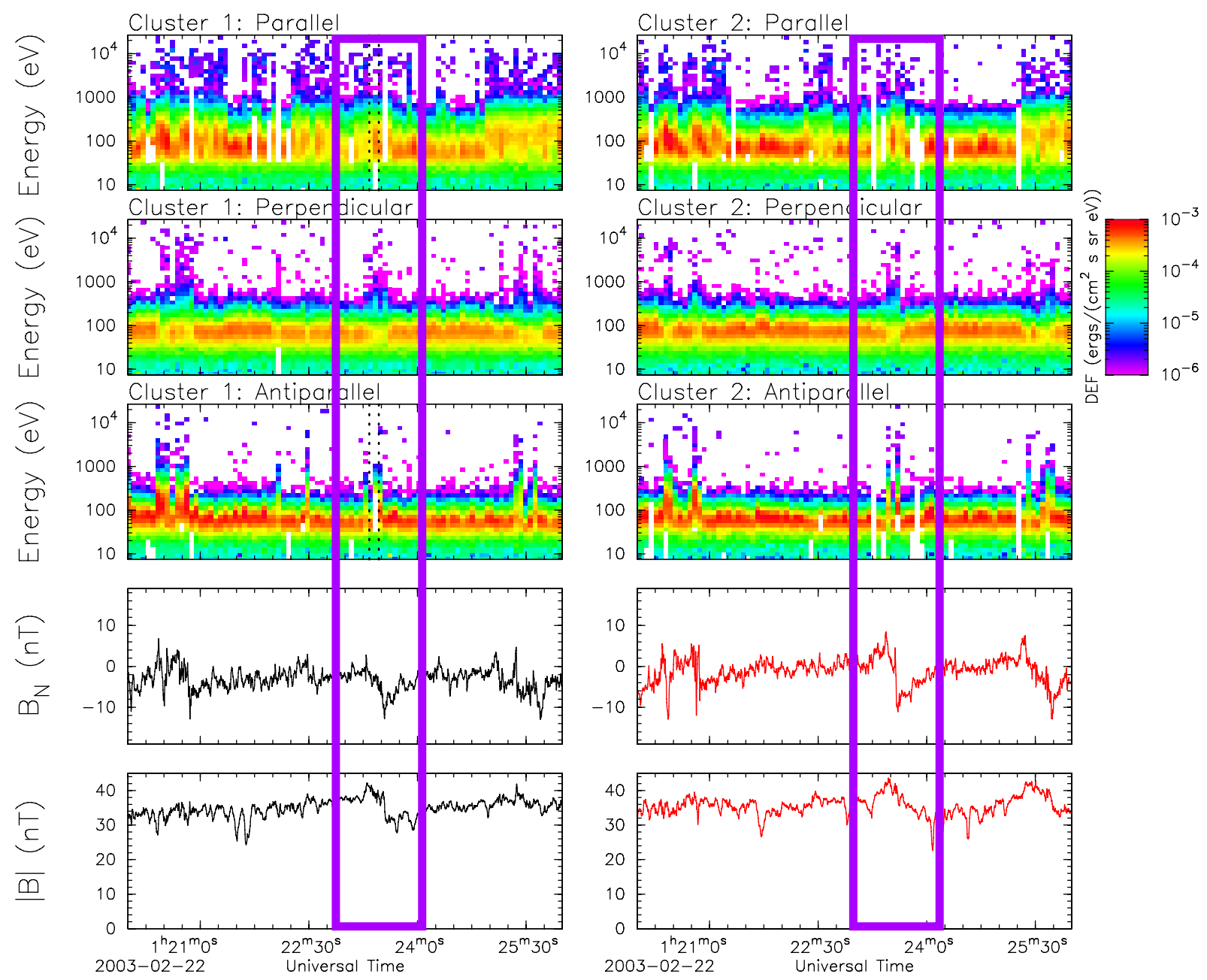

Fig. 11. PEACE electron data from Cluster 1 (left) and Cluster 2 (right) for FTE 161 (22 February 2003, 01:23 UT). Each half of the figure adopts the same format as the Fig. 10a. The dotted lines indicate the slices of the pitch angle distributions shown in Fig. 12.

the magnetic field, and no discernible change to the parallel electron population. Example slices of the pitch angle distributions observed by Cluster 1 immediately before and during the burst of antiparallel-moving electrons are shown in Fig. 12. The two distributions are taken at 01:23:20 and 01:23:28 UT (solid and dashed lines respectively). The times of these two slices are indicated in Fig. 11 by vertical dotted lines in the parallel and antiparallel Cluster 1 spectrograms. Immediately before the antiparallel electron enhancement, the electron distribution exhibits a parallel electron isotropy (solid line). When the energisation of antiparallel electrons is observed (dashed line), there is no significant change to the parallel electron population, although the overall population becomes more isotropic. This antiparallel electron signature is consistent with the result of the Cooling model run in Fig. 7, in which the observed FTE velocity was close to the model velocity for an FTE connected to the Northern Hemisphere $\left(\boldsymbol{V}_{\text {HTN }}\right)$. Similar signatures were observed for other
FTEs in this interval (e.g. at 01:20-01:21 and 01:25 UT in Fig. 11), and were common in the magnetosheath FTEs observed on the 12 and 17 November. However, the electron signatures observed by Cluster 2 for FTE 161 (also shown in Fig. 11) are slightly less clear. Between 01:21 and 01:25 UT, the magnetosheath was generally more isotropic than observed at Cluster 1 (which was closer to the magnetopause). At 01:23 UT, the electron distribution parallel to the magnetic field took a similar form to that observed by Cluster 1 , and there was a sharp and clear signature of energised magnetosheath electrons observed antiparallel to the magnetic field. Where such signatures were observed, they were interpreted as unidirectional electron signatures in the pitch angle in which the signatures were sharper. In the case of FTE 161, this is consistent with the observations made by Cluster 1. If there was not a clear distinction in the "sharpness" of the signatures, they were interpreted as bidirectional. 


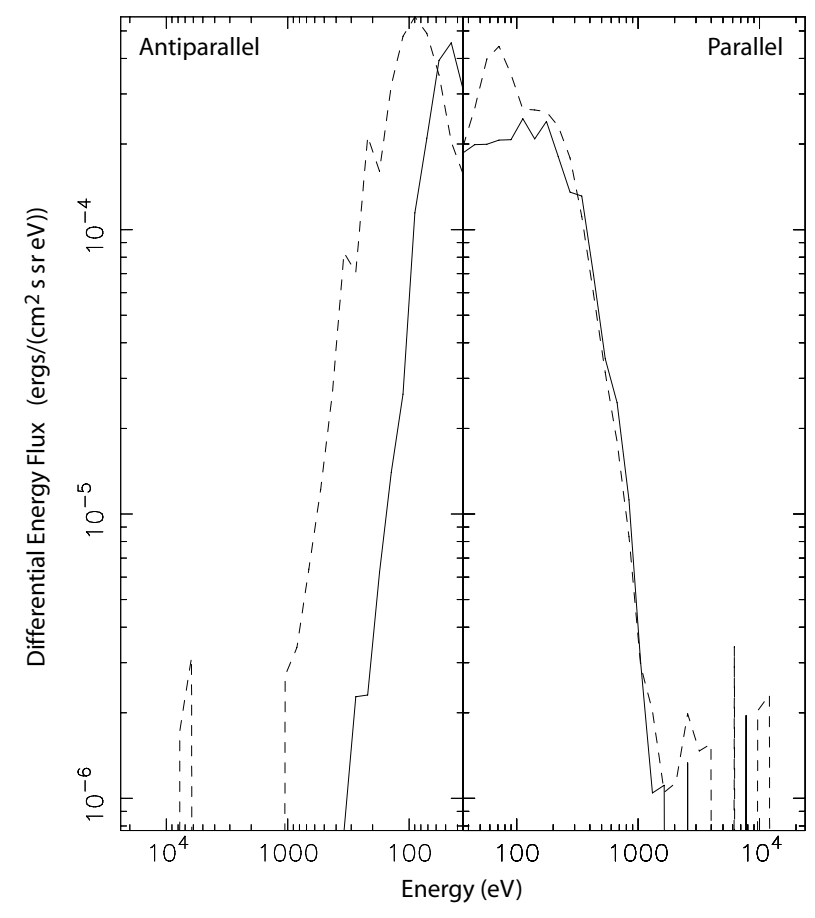

Fig. 12. Two slices of rebinned pitch angle distributions observed by Cluster 1. The left-hand side of the figure represents electrons moving antiparallel to the magnetic field, and the right-hand side represents parallel-moving electrons. The solid line represents the distribution at 01:23:20 UT, shortly before the antiparallel electron signature was observed. The electron distribution exhibited a parallel anisotropy, as is also evident in Fig. 11 (first vertical dotted line). At 01:23:28 UT (inside the antiparallel electron signature, denoted by the second vertical dotted line in Fig. 11), there is no significant change in the distribution of electrons moving parallel to the magnetic field, but there is a clear energisation of the antiparallelmoving electron population.

Only two of the 27 events were associated with no electron signature on any spacecraft for which PEACE data were available. Bidirectional electron signatures were observed for eight magnetosheath FTEs (e.g. FTE 1, Fig. 13).

Twelve of the FTEs in Table 1 exhibit unidirectional electron signatures which verify the results of the Cooling model. However, a further four FTEs have inconsistent electron signatures, demonstrating that the model does not explain the motion of these events. We repeated the calculations in Sect. 5.1 for these four events. In one case (FTE 61, denoted $\S$ in Table 1), there is a small angular difference between the Cooling model value of $\boldsymbol{V}_{\text {HTN }}$ and that which is calculated from observed magnetosheath parameters $\left(5^{\circ}\right)$, but a much larger difference between the values of $\boldsymbol{V}_{\mathrm{HTS}}\left(43^{\circ}\right)$. This is a further example of the sensitivity of the Cooling model when studying the motion of field lines which are connected to the opposite hemisphere from that which would be expected if reconnection took place solely at a tilted subsolar reconnec-
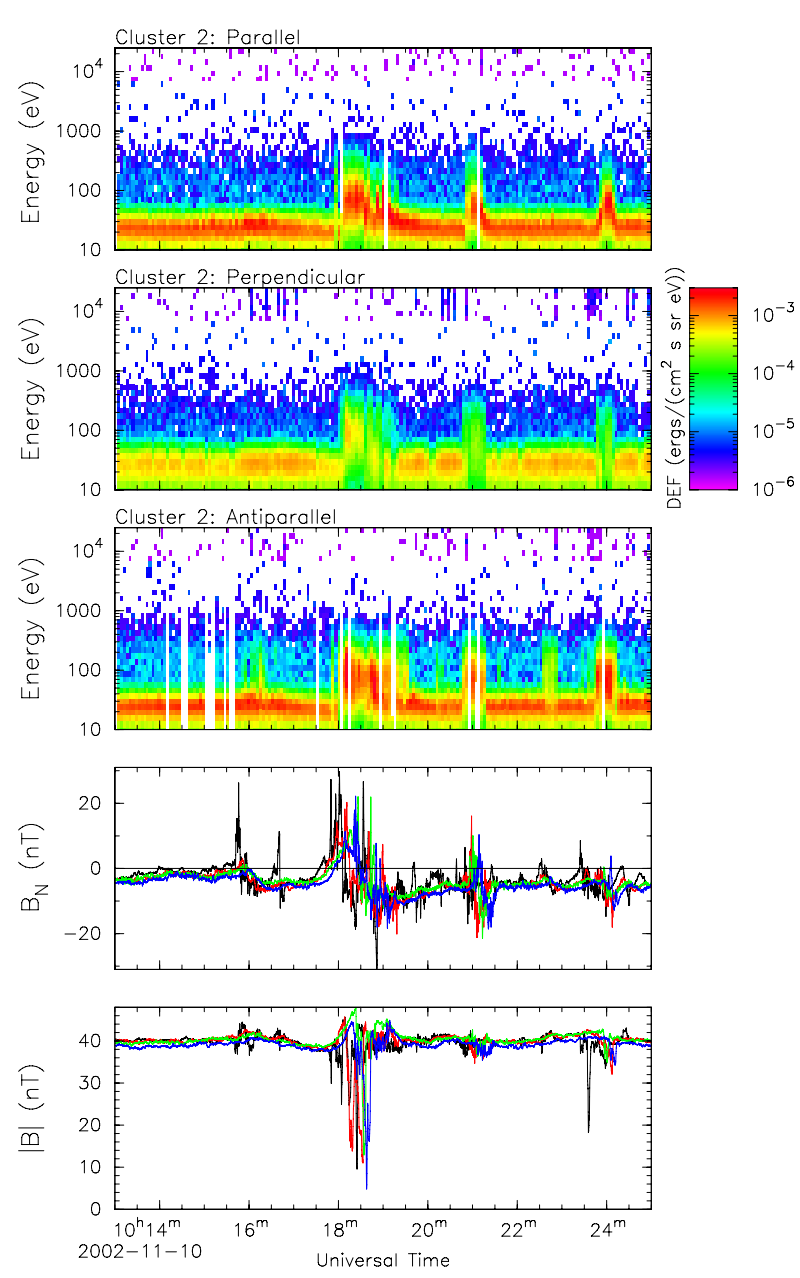

Fig. 13. PEACE electron data from Cluster 2 for FTE 1 (10 November 2002, 10:18 UT). The figure adopts the same format as Fig. 10a. The PEACE instruments observed bidirectional accelerated magnetosheath signatures, possibly due to pitch angle scattering as a result of variations in the magnetic field within the FTE. There are several other FTEs in this plot (e.g. 10:16, 10:17, 10:21, 10:24 UT), which either did not fulfil the criterion for inclusion in the survey carried out by Fear et al. (2005b), or which did not exhibit a clear enough bipolar $B_{N}$ signature on all four spacecraft for multi-spacecraft timing analysis to be attempted.

tion line. The model run and observed velocity of FTE 61 are similar to those shown in Fig. 8, as an equatorward motion is observed as a result of reconnection occuring in a region of super-Alfvénic magnetosheath flow. When this is taken into account, the observed velocity is consistent with $\boldsymbol{V}_{\text {HTS }}$ calculated from the observed magnetosheath parameters, but it is slightly over the $30^{\circ}$ threshold for compatibility with $\boldsymbol{V}_{\text {HTN }}$. The velocity is therefore consistent with the electron signature observed. Furthermore, the other FTEs observed on the same day are all consistent with the Cooling model $\boldsymbol{V}_{\text {HTS }}$ vector (Table 1). 


\subsection{CIS signatures}

The events in Table 1 were also examined for ion signatures using spectrograms from both the HIA and CODIF sensors. The ion signatures of an FTE may be a less reliable indicator of the hemisphere of connection than the electrons, since the larger gyroradii and longer gyroperiods of ions make them less sensitive to quick local variations of the magnetic field. Furthermore, the bulk velocity of magnetosheath ions dominate over their thermal velocity, usually leading to a distinct anisotropy in the magnetosheath ion pitch angles. This contrasts with the magnetosheath electron plasma, where the thermal velocities are usually much greater than the bulk velocities, leading to the observation of an isotropic plasma (although we note that, as in Sect. 6.1, an anisotropy is sometimes observed). Therefore, with the exception of the FTEs which were only observed in the magnetosphere (FTEs 23 and 25), the ion signatures described in Table 1 and discussed below refer to any signatures observed in the $20-30 \mathrm{keV}$ energy range. In the case of FTEs 23 and 25, we refer to the ion signatures in the $100 \mathrm{eV}-10 \mathrm{keV}$ energy range (injected magnetosheath plasma). The CIS PADs were provided by both HIA and CODIF. The CODIF sensor can become saturated in the magnetosheath, but the PADs are still reliable in this energy range as the fluxes are lower.

Two of the FTEs in Table 1 exhibited no identifiable ion signature in either HIA or CODIF data at any of the spacecraft. A further four events had unclear or bidirectional ion signatures. Of those events which exhibited a clear unidirectional ion signature, only ten were consistent with the results of the Cooling model, and eleven were inconsistent. Eight of these had an ion flow direction at which was opposite to the electron signature. The difficulty in interpreting the CIS signatures is illustrated by FTEs 27, 28 and 29, which all occurred within a five minute period. These FTEs are presented in Fig. 14, which shows the PEACE electron signatures parallel, perpendicular and antiparallel to the magnetic field, the omnidirectional proton count rate observed by CODIF, pitch angle distributions for high and low energy protons $(30 \mathrm{keV}>\mathrm{E}>20 \mathrm{keV}$ and $10 \mathrm{keV}>\mathrm{E}>100 \mathrm{eV}$ respectively) and the $B_{N}$ component. Data from both Clusters 1 and 4 are shown. The three FTEs are indicated by magenta boxes. Throughout the interval, there is an antiparallel anisotropy in the magnetosheath electron distribution observed by Cluster 1 (panel e). Both Clusters 1 and 4 observed a peak in the proton count rates at a pitch angle of about $120^{\circ}$, due to the magnetosheath flow. At 14:40, 14:42 and 14:44 UT, all four spacecraft observed bipolar $B_{N}$ signatures, and both Clusters 1 and 4 observed clear bursts of energised magnetosheath electrons moving parallel to the magnetic field (panels a and b), indicating that the FTEs were connected to the Southern Hemisphere. This is consistent with the Cooling model result, as noted in Table 1. Cluster 4 also observed a slight, but more diffuse, signature in electrons moving antiparallel to the magnetic field (panel f), similar to the signatures shown in Fig. 11. This electron population was more persistent at Cluster 1, which was nearer the magnetopause. At the time of each FTE (and at 14:41 UT, when a smaller FTE was observed by Cluster 4), an enhancement of high energy protons was observed moving antiparallel to the magnetic field (panels $\mathrm{i}$ and $\mathrm{j}$ ). These high energy protons are the criterion used to judge the hemisphere of connection from the ion signatures, however they are inconsistent with the hemisphere deduced from the electron signatures. They are also inconsistent with the lower-energy proton signatures (panels k and 1), which show that although the background magnetosheath protons exhibit a $\sim 120^{\circ}$ anisotropy, at the time of each FTE the protons are observed at lower pitch angles, extending towards $0^{\circ}$. Similar signatures were observed by HIA (not shown). Despite the fact that the lower-energy proton pitch angles are usually dominated by the bulk flow in the magnetosheath, they are consistent with the signatures observed in the electron data.

\subsection{RAPID signatures}

We also examined the high-energy particle signatures observed by RAPID. RAPID provides a limited electron pitch angle distribution, but low count rates make this unreliable in the magnetosheath. Therefore, we only examined the events which were observed when the Cluster spacecraft were in a burst mode (indicated in Table 1) as full 3-D distributions were available. A clear enhancement in the differential number flux was observed in only one case and on one spacecraft only (FTE 167, Cluster 3), but there was no clear fieldaligned anisotropy (not shown). This is consistent with the observations made by Daly et al. (1984).

We also examined the high-energy ion signatures for the seven FTEs which occurred when the spacecraft were in burst mode. Two events (FTEs 63 and 65) exhibited no clear high-energy ion signature, but example distributions observed during the remaining five events are shown in Fig. 15. Each panel shows a three dimensional distribution (represented by GSE azimuthal and polar angles) observed during the passage of the FTE. The fluxes are plotted as flow directions; pitch angles indicating flows parallel and antiparallel to the magnetic field are represented by the red circle and red star respectively. Perpendicular pitch angles are represented by a series of purple circles. Each panel represents an accumulation period of 8 spins $(\sim 32 \mathrm{~s})$. Data are unavailable from the central ion heads on each of the RAPID instruments, leading to a data gap in polar angles around $90^{\circ}$.

The first two events (FTEs 61 and 64: Fig. 15, panels a and b) exhibited weak antiparallel high-energy ion signatures, which were also seen in the ion signatures at the top of the CIS energy range (Table 1). However, both FTEs were consistent in velocity with $\boldsymbol{V}_{\text {HTS }}$ (when calculated from observed magnetosheath parameters in the case of FTE 61), and were associated with parallel electron signatures in the PEACE data similar to those seen in Fig. 14. FTE 163 


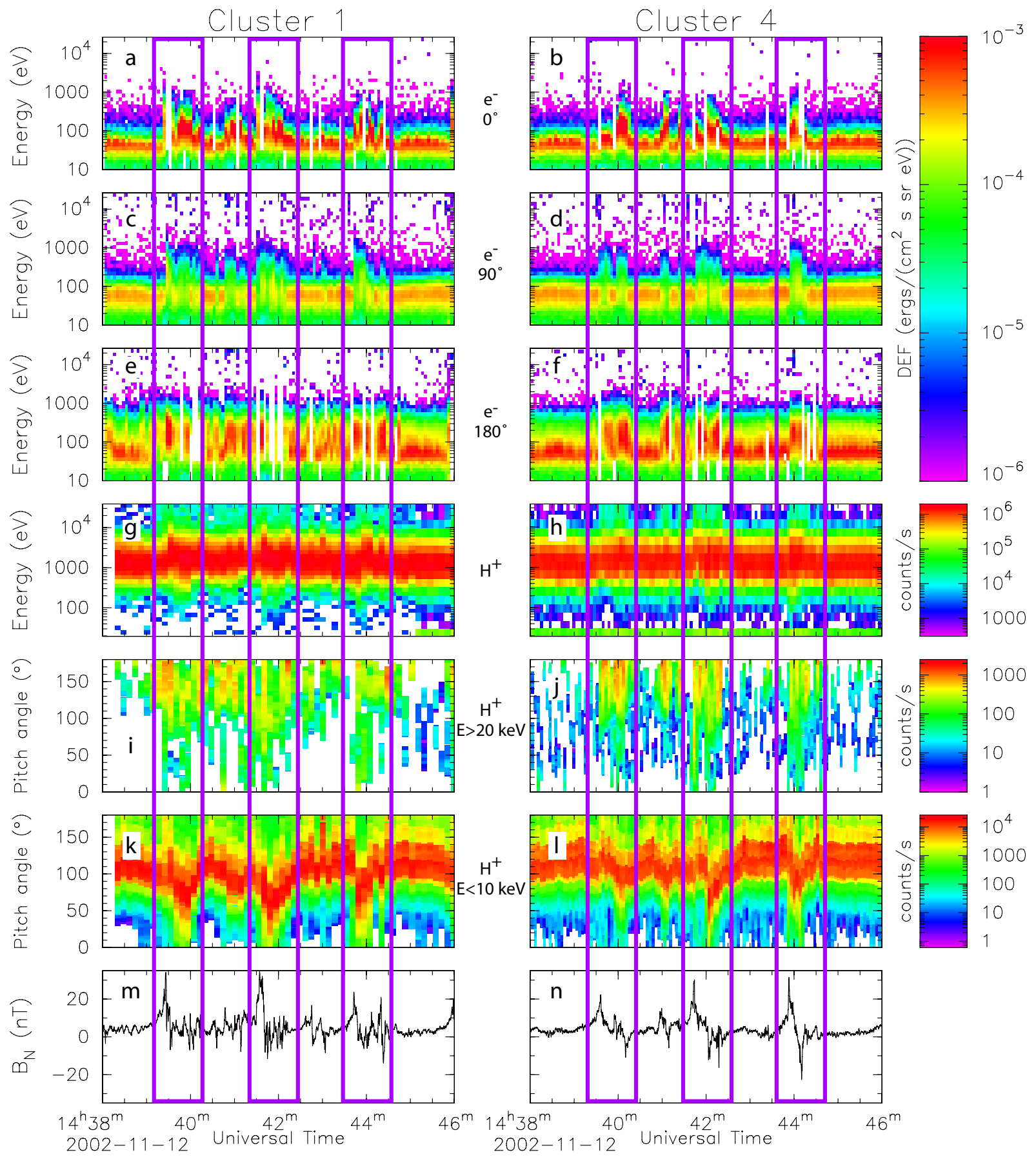

Fig. 14. Plasma and magnetic field data from three FTEs observed on the 12 November 2002. Data from Cluster 1 (left) and Cluster 4 (right) are shown. The panels represent the electron distribution observed by PEACE parallel, perpendicular and antiparallel to the magnetic field (panels a-f), the omnidirectional proton count rate observed by CODIF (panels $\mathbf{g}$ and $\mathbf{h}$ ), the proton pitch angle distribution observed for ions at high energies $(30 \mathrm{keV}>\mathrm{E}>20 \mathrm{keV}$, panels $\mathbf{i}$ and $\mathbf{j})$ and at low energies $(10 \mathrm{keV}>\mathrm{E}>100 \mathrm{eV}$, panels $\mathbf{k}$ and $\mathbf{l})$, and the $B_{N} \mathrm{component}$ (panels $\mathbf{m}$ and $\mathbf{n}$ ). 
(a) FTE 61:Cluster 3 17/11/02 03:23:51 UT
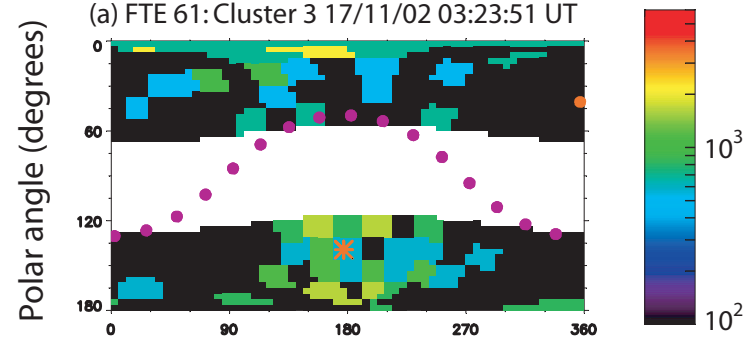

(b) FTE 64: Cluster 4 17/11/02 05:07:53 UT
0 (1)

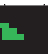

12

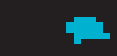

60.
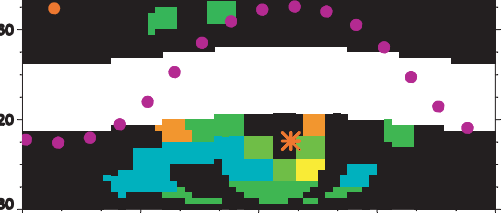

180

(c) FTE 108: Cluster 2 24/12/02 15:50:25 UT

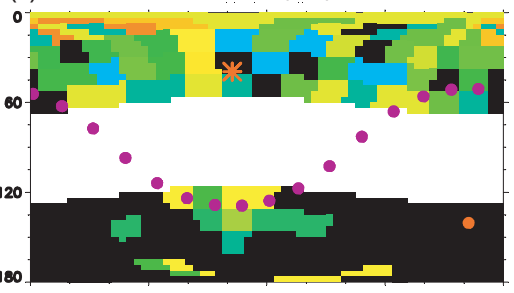

80

(d) FTE 163: Cluster 4 8/3/03 07:07:20 UT

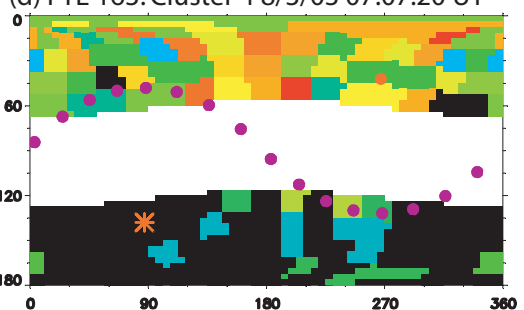

(e) FTE 167: Cluster 3 8/4/03 03:57:41 UT

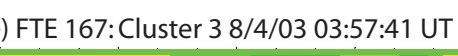

\section{.}

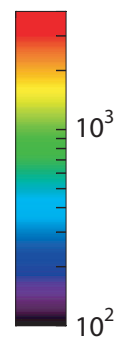$$
\begin{aligned}
& \frac{0}{0} \\
& \frac{d}{0} \\
& \frac{d}{0} \\
& \frac{0}{0} \\
& \frac{1}{0} \\
& \frac{1}{0} \\
& 0
\end{aligned}
$$

120
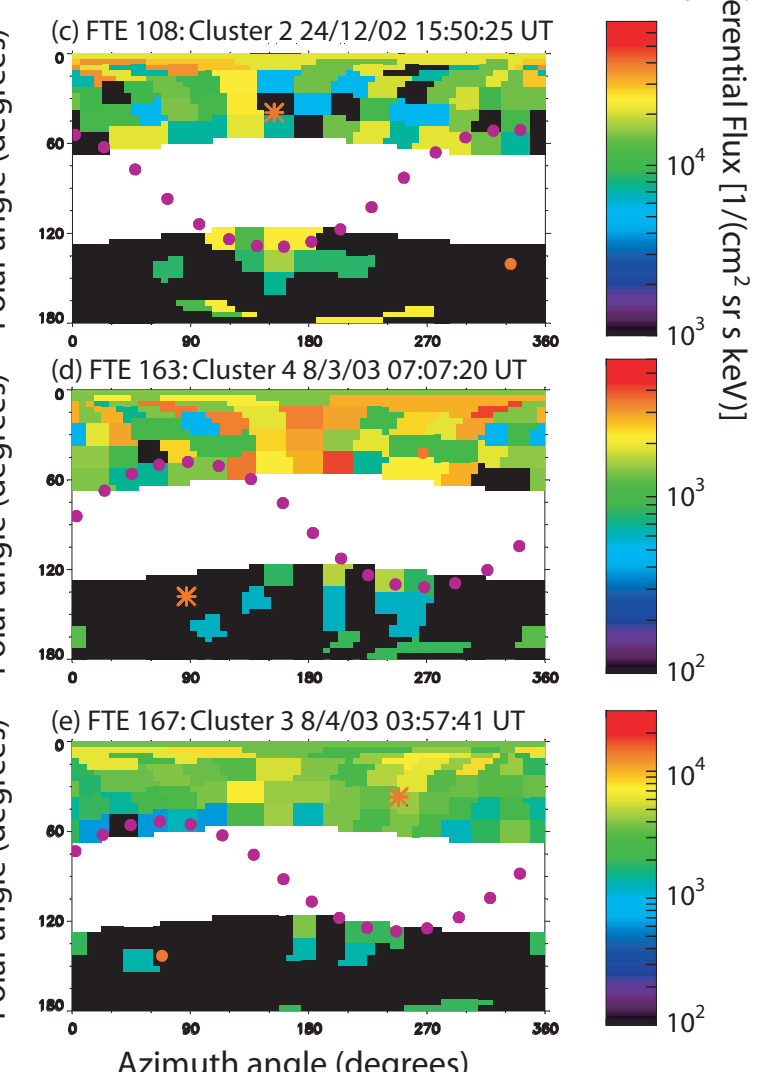

Azimuth angle (degrees)

Fig. 15. RAPID high energy ion distributions for FTEs observed when the Cluster spacecraft were in burst mode. Each panel shows the differential number flux as an azimuthal/polar angle distribution. Parallel and antiparallel pitch angles are indicated by a red circle and star respectively, and perpendicular pitch angles are represented by purple circles. Data are unavailable from the central ion heads, corresponding to polar angles around $90^{\circ}$. (panel d) exhibited a parallel high-energy ion signature that was inconsistent with the hemisphere of connection according to the Cooling model but consistent with the CIS ion signature (although the PEACE electron signature was bidirectional). FTEs 108 and 167 (panels c and e) both had an antiparallel high-energy ion signature, which were both consistent with the PEACE signatures for these events and the connection to the Northern Hemisphere inferred from the Cooling model.

Therefore, all of the events with a clear ion signature in the RAPID data had an anisotropy that was consistent with the highest energy signatures observed by CIS (where a clear directionality was observed in the CIS data). However, the RAPID ion signatures observed in three of the events were inconsistent with the hemisphere of connection implied by the Cooling model. In two of these cases, the RAPID ion signature conflicted with the PEACE signature, which was consistent with the velocity (in the third case, the PEACE signature was bidirectional). In the remaining two cases, the RAPID ion and PEACE electron signatures were both antiparallel to the magnetic field, which was consistent with the results of the Cooling model run.

\section{Discussion}

The Cooling model (Cooling et al., 2001) is a very basic model of reconnected field line motion, which makes several simplifying assumptions, such as a uniformly increasing magnetosheath flow from a subsolar stagnation point. The magnetopause is assumed to be a simple, thin current sheet and a purely rotational discontinuity. Possible local time asymmetries are ignored, as is the effect of reconnection on the bulk flow of the magnetosheath. Longmore et al. (2006) have shown that the rotation between magnetosheath velocities calculated from the Kobel and Flückiger (1994) model used by Cooling et al. (2001) and the observed magnetosheath velocity can be significant (with mean rotations between $5^{\circ}$ and $30^{\circ}$ ). A further caveat is that the Cooling et al. (2001) model, and the calculations by Cowley and Owen (1989) on which it is based, provides the velocity of the reconnected field lines at the point at which they thread the magnetopause. A bundle of reconnected flux may have a different velocity further away from this point.

Nonetheless, we find that the Cooling model usually explains the motion of FTEs at the magnetopause to within an accuracy of $\sim 30^{\circ}$. After excluding three categories of FTEs (those whose motion is poorly defined due to a poor spacecraft tetrahedron and/or weak/unclear signatures, events with a velocity component out of the magnetopause surface by more than $30^{\circ}$, and events with a scale size of less than $\sim 5000 \mathrm{~km}$ ), we are left with a sample of 118 events. 92 of these events $(78 \%)$ are consistent with one of the two de Hoffmann-Teller velocities calculated by the Cooling model in both direction of motion (within $30^{\circ}$ ) and speed 
(observed value between half and double the model value). A further 12 events are consistent in their direction of motion, but not speed (making a total of $88 \%$ which are consistent in direction). The model explains the direction of motion better than an assumption of simple radial flow away from the subsolar point. This indicates that magnetic tension forces remain a significant factor in a the motion of an FTE throughout its existence.

The Cooling model explains the FTE speeds more reliably when the IMF is southward or dominated by $B_{Y}$. If we take only the FTEs with a lagged IMF clock angle magnitude that was greater than $70^{\circ}$, the proportion of FTEs which are consistent with the model in speed and direction is raised slightly $(84 \%)$ whereas there is no change in the proportion which are consistent if the speed criterion is dropped (88\%).

It is not clear why smaller scale events are poorly described by the Cooling model, as multi-spacecraft timing analysis is carried out on a plane defined by the mid-points of each FTE signature rather than on the surface of the structure (which will have a significant curvature on the separation scale of the spacecraft in this season if the FTE scale size is less than $5000 \mathrm{~km}$ ). Furthermore, it is also unclear why some FTEs exhibit a large component of velocity out of the magnetopause plane, since we excluded events which occurred as the spacecraft crossed from the magnetosphere to the magnetosheath or vice versa.

The ambiguity of two model de Hoffmann-Teller velocities (due to a connection to either the Northern or the Southern Hemisphere) can be removed by examining the plasma signature of magnetosheath events. However, several of the magnetosheath FTEs exhibited bidirectional accelerated magnetosheath electron signatures, indicating that it is not always possible to identify the hemisphere of connection of a magnetosheath FTE, even if an electron signature is observed. The electron signature of an FTE in the magnetosheath is largely due to magnetosheath electrons which have been energised at the kink in the reconnected magnetic field lines at the magnetopause. These electrons stream away from the magnetopause, having either been mirrored and energised at the kink, or having been transmitted across the kink, energised, mirrored at low altitudes and been energised a second time as they are transmitted across the kink again back into the magnetosheath. This process alone does not allow a bidirectional electron signature in the magnetosheath, implying that the bidirectionality must be due either to pitchangle scattering on the reconnected field lines in the magnetosheath, reflection of the energised electrons somehow in the magnetosheath (for example, at the bow shock), entry onto some form of closed loop structure, or entry into the magnetosphere in the core of the FTE (e.g. Robert et al., 2005).

Where there was a clear electron anisotropy, it was generally consistent with the hemisphere of connection of the FTE. Out of the 16 events for which there was a clear directionality in the electron signature and which were consistent with one (but not both) of the model FTE velocities in the
Cooling model, 12 exhibited a sense of anisotropy that was consistent with the hemisphere of connection predicted by the Cooling model. In one further case, the hemisphere of connection predicted by a comparison of the observed velocity with Eqs. (6) and (7) reversed if observed magnetosheath parameters were used to evaluate the de Hoffmann-Teller velocities, rather than the Cooling model. When this is taken into account, the electron signatures are consistent with the hemisphere of connection implied by the Cowley and Owen (1989) calculation. This is a consequence of the sensitivity of the model when the Alfvénic part of the motion (due to the magnetic tension in the kinked magnetic field line) is directed oppositely to the magnetosheath flow. This situation arises when reconnection takes place in a region of super-Alfvénic magnetosheath flow (e.g. Fear et al., 2005b). However, when the magnetosheath flow and magnetic tension force are directed in broadly the same direction, the Cooling model results are a lot more stable. Nonetheless, it is advisable to check the model results against the evaluation of Eqs. (6) and (7) when carrying out case studies of FTE motion when magnetosheath observations are available.

It is evident from the examples presented in Fig. 14 that the ion signatures in the energy range observed by CIS are often complicated and are therefore not as good an indicator of the hemisphere of connection as the electron signatures observed by PEACE. Furthermore, as noted by Daly et al. (1984), high-energy electron signatures are also not useful indicators. High-energy electron pitch angle data were only available for seven of the FTEs listed in Table 1, and most did not exhibit a clear signature. One FTE had a relatively clear high-energy electron signature, but this was not coupled with a clear parallel or antiparallel anisotropy. Daly et al. (1984) concluded that the electrons depopulated the opened magnetic field lines too rapidly for most of the escaping magnetospheric electrons to be observed, leaving only a low-level isotropic background. The high-energy ion signatures observed by RAPID were consistent with the highest-energy CIS observations, but were also not as successful an indicator as the PEACE electron observations.

It is worth noting that FTE 161 (Figs. 7 and 11) is a good example of an FTE generated by subsolar component reconnection. The lagged IMF for this event was dominated by $B_{Y}$, although slightly northward. The maximum shear between the model magnetosheath magnetic field and the model geomagnetic field along the path of the model FTE indicated by the red line in Fig. 7 was $83^{\circ}$. The FTE is located relatively close to the model cusp positions; varying the location of the model cusps can significantly alter the shear between the model magnetic fields either side of the magnetopause. However, the spacecraft tetrahedron straddled the magnetopause at the time when the FTE was observed, and so the position of the spacecraft relative to the cusp can be checked. Cluster 3 observed a northward and tailward magnetospheric magnetic field, consistent with the spacecraft being on dayside magnetic field lines near local 
noon. The shear between the geomagnetic field observed by Cluster 3 and the magnetosheath magnetic field observed by the other three spacecraft is $52^{\circ}$; therefore the observations confirm that this event is not consistent with an antiparallel reconnection site. Other events in Table 1 appeared more consistent with a high-shear (antiparallel) reconnection site, but none of these events had clear electron signatures which confirmed the hemisphere of connection.

\section{Conclusions}

We have calculated the velocity of 213 flux transfer events, which were observed under a range of IMF conditions, from the 2002/2003 Cluster magnetopause crossing season. Events with poorly-defined velocities, due to a combination of less-clear $B_{N}$ signatures and a poor spacecraft tetrahedron, were discarded. We find that the model of reconnected field line motion developed by Cooling et al. (2001) explains the motion of the remaining FTEs reasonably well, despite the simplicity of the model. However, there are still two classes of events which are not explained well by the model: FTEs with an apparently significant component of velocity out of the magnetopause surface and FTEs with a scale size less than $\sim 5000 \mathrm{~km}$. Velocities predicted by the model are relatively stable when the drag force exerted by the magnetosheath flow and the magnetic tension force act in broadly the same direction, but are relatively sensitive when these forces oppose each other as is the case for one set of reconnected field lines when reconnection occurs in a region of super-Alfvénic magnetosheath flow. Accelerated magnetosheath electron signatures are usually a good indicator of the hemisphere of connection of FTEs and confirm the accuracy of the model (although bidirectional electron signatures are often observed), but the high-energy ion signatures of magnetosheath FTEs are more complicated and are less useful for this task.

Acknowledgements. The authors thank the PEACE, FGM, CIS and RAPID operations teams for their work on the Cluster data, and the ACE SWEPAM and MAG teams and ACE Science Center for providing the ACE data. R. C. Fear would also like to thank S. J. Schwartz and J. A. Wild for useful comments, and we thank the referees for their suggestions. R. C. Fear was supported by PPARC grant number PPA/G/O/2003/00013. Some data analysis was carried out using the QSAS science analysis system provided by the United Kingdom Cluster Science Centre (Imperial College London and Queen Mary, University of London) supported by The Particle Physics and Astronomy Research Council (PPARC).

Topical Editor I. A. Daglis thanks D. Sibeck and Y. Wang for their help in evaluating this paper.

\section{References}

Anderson, R. R., Harvey, C. C., Hoppe, M. M., Tsurutani, B. T., Eastman, T. E., and Etcheto, J.: Plasma waves near the magnetopause, J. Geophys. Res., 87, 2087-2107, 1982.
Balogh, A., Carr, C. M., Acuña, M. H., Dunlop, M. W., Beek, T. J., Brown, P., Fornaçon, K.-H., Georgescu, E., Glassmeier, K.-H., Harris, J., Musmann, G., Oddy, T., and Schwingenschuh, K.: The Cluster Magnetic Field Investigation: Overview of in-flight performance and initial results, Ann. Geophys., 19, 1207-1217, 2001 ,

http://www.ann-geophys.net/19/1207/2001/.

Berchem, J. and Russell, C. T.: Flux transfer events on the magnetopause - spatial distribution and controlling factors, J. Geophys. Res., 89, 6689-6703, 1984.

Cooling, B. M. A., Owen, C. J., and Schwartz, S. J.: Role of the magnetosheath flow in determining the motion of open flux tubes, J. Geophys. Res., 106, 18 763-18 776, 2001.

Cowley, S. W. H.: Evidence for the Occurrence and Importance of Reconnection between the Earth's Magnetic Field and the Interplanetary Magnetic Field, in: Magnetic Reconnection in Space and Laboratory Plasmas, edited by: Hones, E. W., vol. 30 of Geophysical Monograph, pp. 375-378, AGU, Washington D.C., 1984.

Cowley, S. W. H. and Owen, C. J.: A simple illustrative model of open flux tube motion over the dayside magnetopause, Planet. Space Sci., 37, 1461-1475, 1989.

Daly, P. W., Williams, D. J., Russell, C. T., and Keppler, E.: Particle signature of magnetic flux transfer events at the magnetopause, J. Geophys. Res., 86, 1628-1632, 1981.

Daly, P. W., Rijnbeek, R. P., Sckopke, N., Russell, C. T., and Saunders, M. A.: The distribution of reconnection geometry in flux transfer events using energetic ion, plasma and magnetic data, J. Geophys. Res., 89, 3843-3854, 1984.

Dungey, J. W.: Interplanetary magnetic field and the auroral zones, Phys. Rev. Lett., 6, 48-49, 1961.

Dungey, J. W.: The structure of the exosphere or Adventures in velocity space, in: The Earth's Environment, edited by: DeWitt, C., Hieblot, J., and Lebeau, A., pp. 505-550, Gordon and Breach, New York, 1963.

Dunlop, M. W., Taylor, M. G. G. T., Davies, J. A., Owen, C. J., Pitout, F., Fazakerley, A. N., Pu, Z., H., L., Bogdanova, Y. V., Zong, Q.-G., Shen, C., Nykyri, K., Lavraud, B., Milan, S. E., Phan, T. D., Rème, H., P., E. C., Carr, C. M., Cargill, P., Lockwood, M., and Sonnerup, B.: Coordinated Cluster/Double Star observations of dayside reconnection signatures, Ann. Geophys., 23, 2867-2875, 2005,

http://www.ann-geophys.net/23/2867/2005/.

Elphic, R. C.: Observations of flux transfer events: Are FTEs flux ropes, islands, or surface waves?, in: Physics of Magnetic Flux Ropes, edited by: Russell, C. T., Priest, E. R., and Lee, L. C., vol. 58 of Geophysical Monograph, pp. 455-471, AGU, Washington D.C., 1990.

Elphic, R. C., Baumjohann, W., Cattell, C. A., Lühr, H., and Smith, M. F.: A search for upstream pressure pulses associated with flux transfer events: An AMPTE/ISEE case study, J. Geophys. Res., 99, 13 521-13 527, 1994.

Fear, R. C., Fazakerley, A. N., Owen, C. J., Lahiff, A. D., Lucek, E. A., Balogh, A., Kistler, L. M., Mouikis, C., and Rème, H.: Cluster observations of boundary layer structure and a flux transfer event near the cusp, Ann. Geophys., 23, 2605-2620, 2005a.

Fear, R. C., Fazakerley, A. N., Owen, C. J., and Lucek, E. A.: A survey of flux transfer events observed by Cluster during strongly northward IMF, Geophys. Res. Lett., 32, L18105, doi:10.1029/ 
2005GL023811, 2005b.

Frey, H. U., Phan, T. D., Fuselier, S. A., and Mende, S. B.: Continuous magnetic reconnection at Earth's magnetopause, Nature, 426, 533-537, 2003.

Haerendel, G., Paschmann, G., Sckopke, N., Rosenbauer, H., and Hedgecock, P. C.: The frontside boundary layer of the magnetosphere and the problem of reconnection, J. Geophys. Res., 83, 3195-3216, 1978.

Harvey, C. C.: Spatial gradients and the volumetric tensor, in: Analysis Methods for Multi-Spacecraft Data, edited by Paschmann, G. and Daly, P. W., pp. 307-348, ISSI, 1998.

Hasegawa, H., Sonnerup, B. U. O., Owen, C. J., Klecker, B., Paschmann, G., Balogh, A., and Rème, H.: The structure of flux transfer events recovered from Cluster data, Ann. Geophys., 24, 603-618, 2006,

http://www.ann-geophys.net/24/603/2006/.

de Hoffmann, F. and Teller, E.: Magneto-hydrodynamic shocks, Phys. Rev., 80, 692-703, 1950.

Johnstone, A. D., Alsop, C., Burdge, S., Carter, P. J., Coates, A. J., Coker, A. J., Fazakerley, A. N., Grande, M., Gowen, R. A., Gurgiolo, C., Hancock, B. K., Narheim, B., Preece, A., Sheather, P. H., Winningham, J. D., and Woodliffe, R. D.: PEACE: A Plasma Electron And Current Experiment, Space Sci. Rev., 79, 351-398, 1997.

Kawano, H. and Russell, C. T.: Survey of flux transfer events observed with the ISEE 1 spacecraft: Dependence on the interplanetary magnetic field, J. Geophys. Res., 102, 11 307-11314, doi: 10.1029/97JA00481, 1997a.

Kawano, H. and Russell, C. T.: Cause of postterminator flux transfer events, J. Geophys. Res., 102, 27 029-27 038, doi:10.1029/ 97JA02139, 1997b.

Kawano, H., Kokubun, S., and Takahashi, K.: Survey of transient magnetic field events in the dayside magnetosphere, J. Geophys. Res., 97, 10 677-10 692, 1992.

Kobel, E. and Flückiger, E. O.: A model of the steady state magnetic field in the magnetosheath, J. Geophys. Res., 99, $23617-$ $23622,1994$.

Kuo, H., Russell, C. T., and Le, G.: Statistical studies of flux transfer events, J. Geophys. Res., 100, 3513-3519, 1995.

Lanzerotti, L. J.: Comment on "Solar wind dynamic pressure variations and transient magnetospheric signatures", Geophys. Res. Lett., 16, 1197-1199, 1989.

Lee, L. C. and Fu, Z. F.: A theory of magnetic flux transfer at the Earth's magnetopause, Geophys. Res. Lett., 12, 105-108, 1985.

Lockwood, M.: Flux transfer events at the dayside magnetopause: Transient reconnection or magnetosheath dynamic pressure pulses?, J. Geophys. Res., 96, 5497-5509, 1991.

Longmore, M., Schwartz, S. J., and Lucek, E. A.: Rotation of the magnetic field in Earth's magnetosheath by bulk magnetosheath plasma flow, Ann. Geophys., 24, 339-354, 2006, http://www.ann-geophys.net/24/339/2006/.

McComas, D. J., Bame, S. J., Barker, P., Feldman, W. C., Phillips, J. L., Riley, P., and Griffee, J. W.: Solar Wind Electron Proton Alpha Monitor (SWEPAM) for the Advanced Composition Explorer, Space Sci. Rev., 86, 563-612, 1998.

Nishida, A.: Can random reconnection on the magnetopause produce the low latitude boundary layer?, Geophys. Res. Lett., 16, 227-230, 1989.

Owen, C. J., Fazakerley, A. N., Carter, P. J., Coates, A. J., Krauk- lis, I. C., Szita, S., Taylor, M. G. G. T., Travnicek, P., Watson, G., Wilson, R. J., Balogh, A., and Dunlop, M. W.: Cluster PEACE observations of electrons during magnetospheric flux transfer events, Ann. Geophys., 19, 1509-1522, 2001,

http://www.ann-geophys.net/19/1509/2001/.

Paschmann, G., Haerendel, G., Papamastorakis, I., Sckopke, N., Bame, S. J., Gosling, J. T., and Russell, C. T.: Plasma and magnetic field characteristics of magnetic flux transfer events, J. Geophys. Res., 87, 2159-2168, 1982.

Phan, T., Dunlop, M., Paschmann, G., Klecker, B., Bosqued, J., Rème, H., Balogh, A., Twitty, C., Mozer, F., Carlson, C., Mouikis, C., and Kistler, L.: Cluster observations of continuous reconnection at the magnetopause under steady interplanetary magnetic field conditions, Ann. Geophys., 22, 2355-2367, 2004 , http://www.ann-geophys.net/22/2355/2004/.

Rème, H., Aoustin, C., Bosqued, J. M., Dandouras, I., Lavraud, B., Sauvaud, J. A., Barthe, A., Bouyssou, J., Camus, T., Coeur-Joly, O., Cros, A., Cuvilo, J., Ducay, F., Garbarowitz, Y., Medale, J. L., Penou, E., Perrier, H., Romefort, D., Rouzaud, J., Vallat, C., Alcaydé, D., Jacquey, C., Mazelle, C., D’Uston, C., Möbius, E., Kistler, L. M., Crocker, K., Granoff, M., Mouikis, C., Popecki, M., Vosbury, M., Klecker, B., Hovestadt, D., Kucharek, H., Kuenneth, E., Paschmann, G., Scholer, M., Sckopke, N., Seidenschwang, E., Carlson, C. W., Curtis, D. W., Ingraham, C., Lin, R. P., McFadden, J. P., Parks, G. K., Phan, T., Formisano, V., Amata, E., Bavassano-Cattaneo, M. B., Baldetti, P., Bruno, R., Chionchio, G., di Lellis, A., Marcucci, M. F., Pallocchia, G., Korth, A., Daly, P. W., Graeve, B., Rosenbauer, H., Vasyliunas, V., McCarthy, M., Wilber, M., Eliasson, L., Lundin, R., Olsen, S., Shelley, E. G., Fuselier, S., Ghielmetti, A. G., Lennartsson, W., Escoubet, C. P., Balsiger, H., Friedel, R., Cao, J.-B., Kovrazhkin, R. A., Papamastorakis, I., Pellat, R., Scudder, J., and Sonnerup, B.: First multispacecraft ion measurements in and near the Earth's magnetosphere with the identical Cluster Ion Spectrometry (CIS) experiment, Ann. Geophys., 19, 1303-1354, 2001 , http://www.ann-geophys.net/19/1303/2001/.

Rijnbeek, R. P. and Cowley, S. W. H.: Magnetospheric flux erosion events are flux transfer events, Nature, 309, 135-138, 1984.

Rijnbeek, R. P., Cowley, S. W. H., Southwood, D. J., and Russell, C. T.: Observations of reverse polarity flux transfer events at the Earth's magnetopause, Nature, 300, 23-26, 1982.

Rijnbeek, R. P., Cowley, S. W. H., Southwood, D. J., and Russell, C. T.: A survey of dayside flux transfer events observed by ISEE1 and ISEE-2 magnetometers, J. Geophys. Res., 89, 786-800, 1984.

Robert, P., Lecontel, O., Roux, A., Canu, P., Fontaine, D., Chanteur, G., Bosqued, J., Owen, C. J., Fazakerley, A. N., and Dunlop, M. W.: Study of a Flux Transfer Event with Cluster Spacecraft, in: Proc. Cluster and Double Star Symposium, 5th Anniversary of Cluster in Space, ESA SP-598, 2005.

Roelof, E. C. and Sibeck, D. G.: Magnetopause shape as a bivariate function of interplanetary magnetic field $B_{Z}$ and solar wind dynamic pressure, J. Geophys. Res., 98, 21 421-21 450, 1993.

Russell, C. T. and Elphic, R. C.: Initial ISEE magnetometer results: Magnetopause observations, Space Sci. Rev., 22, 681-715, 1978.

Russell, C. T. and Elphic, R. C.: ISEE observations of flux transfer events at the dayside magnetopause, Geophys. Res. Lett., 6, 33 
36, 1979.

Russell, C. T., Mellott, M. M., Smith, E. J., and King, J. H.: Multiple spacecraft observations of interplanetary shocks: Four spacecraft determination of shock normals, J. Geophys. Res., 88, 4739-4748, 1983.

Russell, C. T., Berchem, J., and Luhmann, J. G.: On the source region of flux transfer events, Adv. Space Res., 5, 363-368, 1985.

Sanny, J., Sibeck, D. G., Venturini, C. C., and Russell, C. T.: A statistical study of transient events in the outer dayside magnetosphere, J. Geophys. Res., 101, 4939-4952, 1996.

Saunders, M. A., Russell, C. T., and Sckopke, N.: Flux transfer events: Scale size and interior structure, Geophys. Res. Lett., 11, 131-134, 1984.

Schield, M. A.: Pressure Balance between Solar Wind and Magnetosphere, J. Geophys. Res., 74, 1275-1286, 1969.

Scholer, M.: Magnetic flux transfer at the magnetopause based on single X-line bursty reconnection, Geophys. Res. Lett., 15, 291294, 1988.

Sckopke, N.: Plasma structure near the low-latitude boundary layer: A rebuttal, J. Geophys. Res., 96, 9815-9820, 1991.

Sibeck, D. G.: A model for the transient magnetospheric response to sudden solar wind dynamic pressure variations, J. Geophys. Res., 95, 3755-3771, 1990.

Sibeck, D. G.: Transient events in the outer magnetosphere: Boundary waves or flux transfer events?, J. Geophys. Res., 97, 40094026, 1992.

Sibeck, D. G. and Newell, P. T.: Pressure-pulse driven surface waves at the magnetopause: A rebuttal, J. Geophys. Res., 100, $21773-21778,1995$.

Sibeck, D. G. and Newell, P. T.: Reply, J. Geophys. Res., 101, 13 351-13 352, 1996.

Sibeck, D. G., Baumjohann, W., Elphic, R. C., Fairfield, D. H., Fennell, J. F., Gail, W. B., Lanzerotti, L. J., Lopez, R. E., Luehr, H., Lui, A. T. Y., MacLennan, C. G., McEntire, R. W., Potemra, T. A., Rosenberg, T. J., and Takahashi, K.: The magnetospheric response to 8-minute period strong-amplitude upstream pressure variations, J. Geophys. Res., 94, 2505-2519, 1989.

Smith, C. W., L'Heureux, J., Ness, N. F., Acuña, M. H., Burlaga, L. F., and Scheifele, J.: The ACE magnetic fields experiment, Space Sci. Rev., 86, 613-632, 1998.

Smith, M. F. and Owen, C. J.: Temperature anisotropies in a magnetospheric FTE, Geophys. Res. Lett., 19, 1907-1910, 1992.

Song, P., Le, G., and Russell, C. T.: Observational differences between flux transfer events and surface waves at the magnetopause, J. Geophys. Res., 99, 2309-2320, 1994.

Song, P., Le, G., and Russell, C. T.: Comment on "Pressure-pulse driven surface waves at the magnetopause: A rebuttal", J. Geophys. Res., 101, 13 349-13 350, 1996.
Sonnerup, B. U. Ö.: On the stress balance in flux transfer events, J. Geophys. Res., 92, 8613-8620, 1987.

Southwood, D. J., Saunders, M. A., Dunlop, M. W., MierJedrzejowicz, W. A. C., and Rijnbeek, R. P.: A survey of flux transfer events recorded by the UKS spacecraft magnetometer, Planet. Space Sci., 34, 1349-1359, 1986.

Southwood, D. J., Farrugia, C. J., and Saunders, M. A.: What are flux transfer events?, Planet. Space Sci., 36, 503-508, 1988.

Spreiter, J. R., Summers, A. L., and Alksne, A. Y.: Hydromagnetic flow around the magnetosphere, Planet. Spa. Sci., 14, 223-253, 1966.

Thompson, S. M., Kivelson, M. G., Khurana, K. K., Balogh, A., Rème, H., Fazakerley, A. N., and Kistler, L. M.: Cluster observations of quasi-periodic impulsive signatures in the dayside northern lobe: High-latitude flux transfer events?, J. Geophys. Res., 109, A02213, doi:10.1029/2003JA010138, 2004.

Wang, Y. L., Elphic, R. C., Lavraud, B., Taylor, M. G. G. T., Birn, J., Raeder, J., Russell, C. T., Kawano, H., Zong, Q.-G., Zhang, H., Zhang, X. X., and Friedel, R. H.: Initial results of high-latitude magnetopause and low-latitude flank flux transfer events from 3 years of Cluster observations, J. Geophys. Res., 110, A11221, doi:10.1029/2005JA011150, 2005.

Wang, Y. L., Elphic, R. C., Lavraud, B., Taylor, M. G. G. T., Birn, J., Russell, C. T., Raeder, J., Kawano, H., and Zhang, X. X.: Dependence of flux transfer events on solar wind conditions from 3 years of Cluster observations, J. Geophys. Res., 111, A04224, doi:10.1029/2005JA011342, 2006.

Wild, J. A., Milan, S. E., Cowley, S. W. H., Bosqued, J. M., Rème, H., Nagai, T., Kokubun, S., Saito, Y., Mukai, T., Davies, J. A., Cooling, B. M. A., Balogh, A., and Daly, P. W.: Simultaneous insitu observations of the signatures of dayside reconnection at the high- and low-latitude magnetopause, Ann. Geophys., 23, 445460, 2005, http://www.ann-geophys.net/23/445/2005/.

Wilken, B., Daly, P. W., Mall, U., Aarsnes, K., Baker, D. N., Belian, R. D., Blake, J. B., Borg, H., Büchner, J., Carter, M., Fennell, J. F., Friedel, R., Fritz, T. A., Gliem, F., Grande, M., Kecskemety, K., Kettmann, G., Korth, A., Livi, S., McKenna-Lawlor, S., Mursula, K., Nikutowski, B., Perry, C. H., Pu, Z. Y., Roeder, J., Reeves, G. D., Sarris, E. T., Sandahl, I., Søraas, F., Woch, J., and Zong, Q.-G.: First results from the RAPID imaging energetic particle spectrometer on board Cluster, Ann. Geophys., 19, 1355-1366, 2001, http://www.ann-geophys.net/19/1355/2001/. 